\title{
Characterizing the suckling behavior by video and 3D- accelerometry in humpback whale calves on a breeding ground
}

\author{
Maevatiana N Ratsimbazafindranahaka ${ }^{\text {Corresp., 1, 2, } 3}$, Chloé Huetz ${ }^{2}$, Aristide Andrianarimisa ${ }^{3}$, Joy S Reidenberg ${ }^{4}$, \\ Anjara Saloma ${ }^{1}$, Olivier Adam ${ }^{2,5}$, Isabelle Charrier ${ }^{2}$ \\ ${ }^{1}$ Association Cétamada, Barachois Sainte Marie, Madagascar \\ 2 Institut des Neurosciences Paris-Saclay, Université Paris-Saclay, CNRS, Saclay, France \\ 3 Département de Zoologie et Biodiversité Animale, Université d'Antananarivo, Antananarivo, Madagascar \\ ${ }^{4}$ Center for Anatomy and Functional Morphology, Icahn School of Medicine at Mount Sinai, New York, United States \\ 5 Institut Jean Le Rond d'Alembert, Sorbonne Université, Paris, France \\ Corresponding Author: Maevatiana N Ratsimbazafindranahaka \\ Email address: maevatiana.ratsimbazafindranahaka@universite-paris-saclay.fr
}

Getting maternal milk through nursing is vital for all newborn mammals. Despite its importance, nursing has been poorly documented in humpback whales (Megaptera novaeangliae). Nursing is difficult to observe underwater without disturbing the whales and is usually impossible to observe from a ship. We attempted to observe nursing from the calf's perspective by placing CATS cam tags on three humpback whale calves in the Sainte Marie channel, Madagascar, Indian Ocean, during the breeding seasons. CATS cam tags are animal-borne multi-sensor tags equipped with a video camera, a hydrophone and several auxiliary sensors (including a 3-axis accelerometer, a 3-axis magnetometer, and a depth sensor). The use of multi-sensor tags minimized potential disturbance from human presence. A total of 10.52 hours of video recordings were collected with the corresponding auxiliary data. Video recordings were manually analyzed and correlated with the auxiliary data, allowing us to extract different kinematic features including the depth rate (DR), speed, Fluke Stroke Rate (FSR), Overall Body Dynamic Acceleration (ODBA), pitch, roll and roll rate. We found that suckling events lasted $18.8 \pm 8.8 \mathrm{~s}$ on average $(N=34)$ and were performed mostly during dives. Suckling events represented $1.7 \%$ of the total observation time. During suckling, the calves were visually estimated to be at a $30-45^{\circ}$ pitch angle relative to the midline of their mother's body and were always observed rolling either to the right or to the left. In our auxiliary dataset, we confirmed that suckling behavior was primarily characterized by a high average absolute roll and additionally we also found that it was likely characterized by a high average FSR and a low average speed. Kinematic features were used for supervised machine learning in order to subsequently detect suckling behavior automatically. Our study is a proof of method on which future PeerJ reviewing PDF | (2021:10:66429:1:1:NEW 11 Jan 2022) 
investigations can build upon. It opens new opportunities for further investigation of suckling behavior in humpback whales and the baleen whale species. 
1 Characterizing the suckling behavior by video and 3D-

2 accelerometry in Humpback whale calves on a

3 breeding ground

\section{Corresponding Author:}

Maevatiana Nokoloina Ratsimbazafindranahaka ${ }^{1,2,3}$

Institut des Neurosciences Paris-Saclay (NeuroPSI), UMR 9197 CNRS

Campus CEA Saclay, 151 route de la Rotonde, 91400 Saclay, FRANCE

Email address: maevatiana.ratsimbazafindranahaka@universite-paris-saclay.fr

\section{Abstract}

Getting maternal milk through nursing is vital for all newborn mammals. Despite its importance, nursing has been poorly documented in humpback whales (Megaptera novaeangliae). Nursing is difficult to observe underwater without disturbing the whales and is usually impossible to observe from a ship. We attempted to observe nursing from the calf's perspective by placing CATS cam tags on three humpback whale calves in the Sainte Marie channel, Madagascar, Indian Ocean, during the breeding seasons. CATS cam tags are animal-borne multi-sensor tags equipped with a video camera, a hydrophone, and several auxiliary sensors (including a 3-axis accelerometer, a 3-axis magnetometer, and a depth sensor). The use of multi-sensor tags minimized potential disturbance from human presence. A total of 10.52 hours of video recordings were collected with the corresponding auxiliary data. Video recordings were manually analyzed and correlated with the auxiliary data, allowing us to extract different kinematic features including the depth rate (DR), speed, Fluke Stroke Rate (FSR), Overall Body Dynamic Acceleration (ODBA), pitch, roll, and roll rate. We found that suckling events lasted $18.8 \pm 8.8 \mathrm{~s}$ on average $(N=34)$ and were performed mostly during dives. Suckling events represented $1.7 \%$ of the total observation time. During suckling, the calves were visually estimated to be at a $30-45^{\circ}$ pitch angle relative to the midline of their mother's body and were always observed rolling either to the right or to the left. In our auxiliary dataset, we confirmed 
40 that suckling behavior was primarily characterized by a high average absolute roll and

41

42

43

44

45

46

47

48

49

50

51

52

53

54

55

56

57

58

59

60

61

62

63

64

65

66

67

68

69

70

71

72

73

74

75

76

77

78

79

80

81

82

83

additionally we also found that it was likely characterized by a high average FSR and a low average speed. Kinematic features were used for supervised machine learning in order to subsequently detect suckling behavior automatically. Our study is a proof of method on which future investigations can build upon. It opens new opportunities for further investigation of suckling behavior in humpback whales and the baleen whale species.

\section{Introduction}

Female mammals feed their offspring with maternal milk to ensure their progeny's development and survival during the early dependent life stages of the young (Pond, 1977; Smith, 1977; Daly, 1979; Gittleman \& Thompson, 1988; Balshine, 2012). The maternal behavior associated to the transfer of milk to the offspring is referred to as 'nursing.' From the offspring's perspective, this behavior can be referred to as 'suckling,' i.e., to obtain milk from the mammary gland (breast) through the mammary papilla (nipple or teat) (Hall, Hudson \& Brake, 1988). Suckling can involve an active role of mouth movements from the young to stimulate nipple erection, compress the breast's lactiferous sinuses, and generate negative pressure (suction) along with a pulling motion on the teat in order to withdraw the milk. Alternatively, suckling can involve a passive role in which milk is squirted into the open mouth with no stimulating movements of the offspring's mouth.

Nursing behavior is an integral part of mammalian reproductive behavior (Gittleman \& Thompson, 1988). The duration of lactation (the period of time during which milk can be produced; Hall, Hudson \& Brake, 1988) and the frequency of nursing vary greatly among mammals and are related to the developmental characteristics of the young and their environmental constraints (Oftedal, Boness \& Tedman, 1987; Oftedal, 1993).

Whale breasts are positioned ventro-laterally on either side of the midline genital slit (opening of the vestibule vagina). The location of each breast is marked by a short mammary slit in the overlying blubber through which the nipple can be extruded for nursing. The mammary glandular tissue is located deep to the overlying blubber layer, thus providing a hydrodynamically streamlined outline even when the whale's breasts are engorged with milk during lactation. Lactation in baleen whales typically occurs initially in the winter, when calves are born (Lockyer, 1984; Oftedal, 1993; Bannister, 2018). As most baleen whales migrate, lactation that begins on the calving area continue during the migration to the feeding area (Lockyer, 1984; Oftedal, 1993; Bannister, 2018).

Humpback whales (Megaptera novaeangliae), the subject of this study, are migratory cetaceans. They move between high-latitude (closer to the poles) feeding grounds and lowlatitude (closer to the equator) breeding/calving grounds (Clapham, 2018). Similar to other baleen whales, their nursing strategy is constrained by a prolonged fasting of the mother during lactating period, related to the migratory pattern (Oftedal, 1993). A young humpback whale (calf) feeds exclusively on maternal milk during its first six months of life (Clapham, 2018). The energy intake from the milk is crucial in ensuring rapid growth (Lockyer, 1984) and therefore allowing the calf to migrate to the feeding area in cold waters alongside its mother (Lockyer, 1984; Corkeron \& Connor, 1999; Braithwaite, Meeuwig \& Hipsey, 2015). Mother-calf pairs make slower progress compared to groups not accompanied by calf in part due to presumed slower swimming or frequent pauses that allow the calf to suckle (Noad \& Cato, 2007). After its first six months of life, the calf starts to feed on prey but continues to get nutrition from milk

Peer] reviewing PDF | (2021:10:66429:1:1:NEW 11 Jan 2022) 
84

85

86

87

88

89

90

91

92

93

94

95

96

97

98

99

100

101

102

103

104

105

106

107

108

109

110

111

112

113

114

115

116

117

118

119

120

121

122

123

124

125

126

127

128

until complete weaning at 10 to 12 months old (Chittleborough, 1958; Clapham, 2018). The weaning coincides with the mother returning to the calving area. Although early weaning on the feeding grounds may also occur (Baraff \& Weinrich, 1993; Steiger \& Calambokidis, 2000).

Despite its importance, nursing (and suckling) behavior has been poorly documented in humpback whales because it is difficult to observe with accuracy and certainty. Indeed, nursing can occur at varying depths from the sub-surface $(<5 \mathrm{~m})$ to deep dives (up to $64 \mathrm{~m}$ ) as suggested by camera-observed nursing events in feeding areas (Tackaberry et al., 2020). The earlier descriptions of nursing behavior in humpback whales were mostly based on limited surface and sub-surface observations of stationary or slow moving whales and relied mainly on the positioning of the calf (Glockner \& Venus, 1983; Glockner-Ferrari \& Ferrari, 1985; Clapham \& Mayo, 1987; Morete et al., 2003; Videsen et al., 2017). Clapham and Mayo (1987) for example described nursing as an event between two calf's successive breaths on either sides of the mother's caudal peduncle when the mother is stationary at the surface and during which the calf is beneath the mother. Videsen et al. (2017) defined nursing events by associating them with the occurrence of 'peduncle dives,' defined as repeated dives during which the calf remained submerged beneath the mother's caudal peduncle.

Nevertheless, from these different studies, various nursing modalities have been advanced. While nursing, the mother is either stationary or swimming very slowly at a depth around 10-15 m (Glockner-Ferrari \& Ferrari, 1985), or occasionally at the surface with her tail in the air (Morete et al., 2003). The calf is typically positioned vertically (head up, tail down) beneath the mother (Glockner \& Venus, 1983). Videsen et al. (2017) suggested that to initiate suckling, the calf uses tactile stimuli on the mother rather than vocalizations. Tactile stimulation likely results in an autonomic reflex causing extrusion of the nipple. The calf can then grasp the nipple with its tongue. From an underwater video footage of suckling humpback whale calf recorded in the tropical waters of Tonga, South Pacific Ocean, by Scott Portelli (2021, pers. Comm.), it has been observed that the whale calf has an unusual tongue that is lined with lingual papillae (fringes) that may help the tongue grasp the nipple, and may even seal the tongue into a tube that facilitates transferring milk into the mouth.

Underwater video is the best method for studying the nursing and suckling behaviors of whales. Various solutions are possible. One option is to use a video camera from a surface platform (boat or kayak) but it has two major drawbacks: 1) it is necessary to get very close to the whales and potentially interfere with their behavior (harassment); 2) visual contact is lost when the whales dive deeply, even when the water is very clear. Another option is to use an underwater drone (Remotely Operated underwater Vehicle - ROV or Autonomous Underwater Vehicle - AUV, Butcher et al., 2021). ROVs and AUVs have potential but presently vehicles are not yet agile enough to track whales. They are too slow and difficult to manoeuver, with ROV's also being limited by cable length. Alternatively, underwater video can be obtained during close approaches by breath-holding or scuba divers. However, this is only possible when the whales are stationary or swimming very slowly as it would be impossible for a diver to keep pace with traveling whales. Additionally, the presence of divers in close proximity may disturb the whales and habituation is not a desirable tactic. Scott Portelli (2021, pers. Comm.), Zoidis and LomacMacNair (2017) successfully video documented humpback whale suckling behavior thanks to breath-holding divers. In Zoidis and Lomac-MacNair (2017), the recording of milk clouds in the water column allowed the confirmation of the occurrence of suckling. However, only few events could be recorded ( $N=5$ from four mother-calf pairs). 
The development of animal-borne multi-sensor tags such as Customized Animal Tracking Solutions (CATS) cam tags provided a way for remote and accurate recording of whale behavior (Cade et al., 2016). Bio-loggers can be equipped with a suite of sensors including cameras, accelerometers, gyroscopes, magnetometers, pressure (depth) sensor, temperature sensor, light sensor, and hydrophone. CATS cam tags are particularly well suited to study suckling behavior since in addition to kinematic sensors they also allow the recording of video directly from the calf's perspective and thus allow confident confirmation of suckling events. Such devices were used by Tackabery et al. (2020) to accurately describe the suckling behavior of humpback whale calves on feeding areas. One disadvantage of using an animal-borne tag is the potential stress generated during the tag attachment phase. The deployment must be done by experienced operators following a strict protocol (slow speed approach, brief deployment time, etc.) to minimizes the disturbance (Stimpert et al., 2012; Saloma, 2018).

In this study, we characterized the suckling behavior of $<3$ months old humpback whale calves on their calving ground using CATS cam tags. We aimed to describe in detail: 1) how and at what depth the suckling is performed, its duration, and its frequency; 2) the behavioral signatures of suckling events using accelerometry and depth data; and 3) whether the different kinematic features extracted from only two sensors (i.e., 3-axis accelerometer and depth sensor) among all the available sensors in the CATS cam tags were sufficient for a supervised machine learning algorithm to automatically detect suckling behavior. Supervised machine learning is a type of machine learning in which an algorithm learns from labelled datasets to classify unforeseen data. Such classification technique would open the opportunity to detect suckling events from data collected by tags without video camera such as Acousonde or Dtags or when visual observations are not good enough (e.g., during night time or in water with poor visibility).

\section{Materials \& Methods}

\section{Study area}

The study occurred in the Indian Ocean along coast of Madagascar, specifically in the Sainte Marie channel. The channel is located between the Sainte Marie Island (between latitudes $17^{\circ} 19^{\prime}$ and $16^{\circ} 42^{\prime}$ South, and longitudes $49^{\circ} 48^{\prime}$ and $50^{\circ} 01^{\prime}$ East) and the east coast of Madagascar's mainland. The channel is approximately $60 \mathrm{~km}$ long and $7-30 \mathrm{~km}$ wide. It is relatively shallow: its average depth is about $35 \mathrm{~m}$ and the maximal depth is $60 \mathrm{~m}$ (Trudelle et al., 2016). The tag deployments were conducted as part of an ongoing study on humpback whale mother-calf interactions during the calving season and occurred between August and September of 2018 and 2019.

\section{Tag specifications}

We used CATS cam tags to investigate humpback whale suckling behavior. CATS cam tags are small and lightweight $(\sim 500 \mathrm{~g})$ non-invasive animal-borne multi-sensor tags attached via suction cups. They contain six auxiliary sensors (3-axis accelerometer, 3-axis gyroscope, 3-axis magnetometer, pressure/depth sensor, temperature sensor, and light sensor), a hydrophone, and an HD video camera $\left(100^{\circ}\right.$ Field of view). A VHF transmitter (ATS F1835B) attached on the tag allows tracking for tag retrieval. The sampling frequency was set at $10 \mathrm{~Hz}$ for the magnetometer, gyroscope, depth, and temperature sensors. Accelerometer sampling rate was set at $400 \mathrm{~Hz}$ in 2018 and at $800 \mathrm{~Hz}$ in 2019. Accelerometer data from 2019 were however always downsampled to $400 \mathrm{~Hz}$ in subsequent analysis for a matter of consistency. The camera recorded videos with a $1280 \times 720$-pixel resolution at 30 frames per second in 2018 and with a $1920 \times 1072$-pixel 
173 resolution at 30 frames per second in 2019 . The hydrophone recorded sound at a $48 \mathrm{kHz}$ 174 sampling rate in 2018 and at a $24 \mathrm{kHz}$ sampling rate in 2019 (16-bit resolution). Two individual

175 CATS cam tags were used in our study (designated as Cats1 and Cats2 hereafter).

\section{Tagging procedures}

177 CATS cam tags were deployed on calves accompanied by their mother from a $6.40 \mathrm{~m}$ rigid motor boat using a 5-m carbon-fiber pole. Deployment were performed by researchers experienced in successfully approaching mother-calf pairs with minimal disturbance. The tags were placed on the back, near the dorsal fin of the animal. Calves were tagged using one of the two approaches described in Stimpert et al. (2012) and in Saloma (2018) in order to minimize disturbance to the mother-calf pair. Tagging efforts were terminated if the pair displayed avoidance behavior or if the calf was not successfully tagged within 30 min. Immediate behavioral response of the animals to tagging was recorded as in Stimpert et al. (2012). All mother-calf pairs were photo-identified to avoid double-sampling within the calving season. We attributed a relative age to each tagged calf depending on the angle of furl of the dorsal fin (neonate versus non-neonate, Cartwright \& Sullivan, 2009; Faria et al., 2013; Saloma, 2018).

Tagged animals were not followed after tag deployment to avoid any further disturbance of their behavior. After tag deployment or an aborted attempt, the boat slowly moved away in the opposite direction of the mother-calf pair. The tag was retrieved after few hours or the following day, when it detached itself from the animal (usually as a consequence of rubbing against the mother, surface active behavior, etc.). The VHF tag emitted a continuous signal, facilitating retrieval. All methods and approaches were carried out in accordance with relevant guidelines and regulations in force in Madagascar and were approved by the Ministry of Fisheries Resources, Madagascar, under the national research and collect permits \#28/18MRHP/SG/DGRHP and \#36/19-MAEP/SG/DGPA. This present study complies with the European Union Directive on the Protection of Animals Used for Scientific Purposes (EU Directive 2010/63/EU) and also with current Malagasy laws.

199

200

201

202

203

204

205

206

207

208

209

210

211

212

213

214

215

216

217

\section{Sensor data processing}

Data from all sensors were downloaded as CSV files and imported into MATLAB (Mathworks) using dedicated scripts (CATS Matlab toolkit, https://github.com/wgough/CATS-MethodsMaterials). Raw accelerometer data were downsampled to obtain a common sampling rate of 10 $\mathrm{Hz}$ across all sensors and depth data (in meters) was smoothed with a $0.5 \mathrm{~s}$ running median filter. In all computation described hereafter the output sampling frequency was of $10 \mathrm{~Hz}$. Sensors reading were rotated to match the calf's orientation frame using established methods and raw animal pitch and roll (in degree) were then calculated (Johnson \& Tyack, 2003; Cade et al., 2016; Tackaberry et al., 2020). To obtain pitch and roll data representing only the body posture and without fluke stroke signal, we low-pass filtered the raw pitch and roll data $(0.2 \mathrm{~Hz}$ low-pass filter) as in Simon et al. (2012). On the other hand, we used band-pass filtered raw pitch (0.2-1 $\mathrm{Hz}$ band-pass filter) to obtain the fluke stroke pattern, similar to Simon et al. (2012). Stroking was identified when the band-pass filtered pitch passed from below $-3^{\circ}$ to above $+3^{\circ}$ or vice versa within $6.5 \mathrm{~s}$. The used thresholds were determined by visual inspection of the accelerometry plots (Iwata et al., 2021). From the identified fluke strokes, the Fluke Stroke Rate (FSR, in Hertz) was calculated on the basis of half-strokes (López et al., 2015). Animal forward speed (speed hereafter, in meter/second) was determined using the tag jiggle recorded in the original high frequency accelerometer data (Cade et al., 2017). Speed was smoothed with a $0.5 \mathrm{~s}$ running mean filter. In addition, we calculated the Overall Dynamic Body Acceleration (ODBA, 
218 in $\mathrm{G}$ and converted to meter/second ${ }^{2}$ ) as in Wilson et al. (2006), the roll rate (in degree/second), 219 and the depth rate (in meter/second) using custom scripts and the Animal Tag toolbox

220 (http://www.animaltags.org).

221

222

223

224

225

226

227

Depth data helped determine various diving or surface activity phases. We defined diving as any submergence to a depth of $>10 \mathrm{~m}$ (Stimpert et al., 2012; Saloma, 2018). Dives were further divided into three phases: descent, bottom, and ascent phase. As individual dives could include stops at various depths, the bottom phase was defined as the segment at $>85 \%$ of the maximal dive depth for a dive (Stimpert et al., 2012). The descent phase was defined as the segment starting at the surface that immediately preceded the bottom phase. Inversely, the ascent phase was defined as the segment that follows directly the bottom phase and ends at the surface.

\section{Video data analysis and suckling data extraction}

229 We identified and labelled suckling events from the video files using the Behavioral Observation Research Interactive Software (BORIS; Friard \& Gamba, 2016). The corresponding depth data was displayed concurrent with the video. We defined a suckling event as a period during which the tip of the calf's snout continuously touched $(>2 \mathrm{~s})$ the mammary slit of the mother and a milk cloud (see video clip in https://www.youtube.com/watch? $\mathrm{v}=\mathrm{U}$ cyCgiCieFk), even in low density, was observed in the water during the event or upon release of contact (Tackaberry et al.,

235

236

237

238

239

240

241

242 2020). For each suckling event, we calculated its duration and extracted its corresponding activity phase (descent, bottom, ascent, or surface), together with the average depth, depth rate, speed, FSR, ODBA pitch, roll, and roll rate. In addition to suckling events identification, we estimated the proportion of time each calf was in close proximity under its mother (mother visible above the calf) without suckling. Furthermore, we also counted the number of suckling dives during which one or several confirmed suckling events were recorded and the number of non-suckling dives during which the calf is observed staying at least $5 \mathrm{~s}$ under the mother, in close proximity and without any suckling event.

\section{Comparison of suckling with non-suckling segments}

A reference data set for comparison is needed to contrast and identify the behavioral signatures unique to suckling events. To generate such comparison data, we divided our dataset from each deployment into non-overlapping segments of $20 \mathrm{~s}$ (duration comparable to suckling events). Then we selected 10 random segments that did not match to any suckling event for each activity phase in which suckling occurred, similar to Tackaberry et al. (2020). These selected segments, referred as 'non-suckling segments,' were analyzed for the same characteristics as suckling events (see above). To assess if there was a significant difference between suckling events and non-suckling segments, we used a linear mixed-effects models (LMMs) estimated in R using REML (Restricted Maximum Likelihood) that included the suckling status (suckling versus nonsuckling) and the activity phase as fixed effects, and individuals as random effect (Tackaberry et al., 2020). In the models, we considered the following response variables: average depth rate, average speed, average FSR, average ODBA, average pitch, absolute average roll, and average roll rate. Absolute value has been used for roll to emphasize any deviation from zero. The model's reference levels (intercept) corresponded to bottom non-suckling (non-suckling segment occurring at the bottom phase of dive). The LMMs were followed by Tukey's post-hoc multiple comparison test using the R package emmeans (Lenth et al., 2018). Statistical significance level was set to $\alpha=0.05$. 


\section{Testing automatic identification of suckling behavior using supervised machine 262 learning}

263 Data preparation

264 For the supervised machine learning process, as the goal was to identify automatically suckling 265 and non-suckling in non-labelled data, the entire dataset was included in the analysis (i.e., data 266 with video from tag on to tag off). For each deployment, we split the dataset into $2 \mathrm{~s}$ nonoverlapping blocks (windows) and then determined which behavioral period each block fell under (suckling or non-suckling period). Two classes were thus considered: (1) 'suckling' and (2) 'non-suckling.' Each block falling under both suckling and non-suckling period (i.e. transition to or from a suckling period) was labelled as 'suckling' only if $3 / 5$ of its duration fell under suckling period. Otherwise, it was labelled as 'non-suckling.' For each block, we computed 43 features: mean, minimum, maximum, variance, skewness and kurtosis for depth, depth rate, speed, ODBA, pitch, roll, and roll rate, and finally mean for FSR. Indeed, as suggested in Ladds et al. (2016), a great number of summary statistics may help in detecting subtle differences between classes.

Similar to Jeantet et al. (2020), we segmented the data prior to machine learning implementation. We excluded blocks too close to the surface $(<1.5 \mathrm{~m}$ depth) or of high speed activities $\left(>2 \mathrm{~m} \mathrm{~s}^{-1}\right)$. This exclusion helped in partially removing noise from the data and also in reducing class imbalance. As suggested by observations on a feeding ground, suckling events occur mostly at depth (Tackaberry et al., 2020). Also, given the size of the mothers (>11 $\mathrm{m}$ in length and $>2 \mathrm{~m}$ diameter at the umbilical section level in adult whales, as determined by estimates in the field), the generally observed nursing configuration (calf generally below the mother), and speed during nursing (Zoidis \& Lomac-MacNair, 2017; Tackaberry et al., 2020), we were confident that no suckling events would be recorded at $<1.5 \mathrm{~m}$ depth or at $>2 \mathrm{~m} \mathrm{~s}^{-1}$ speed. We checked the validity of these threshold assumptions in our data to ensure that indeed no suckling events were removed partially or entirely. Validating these parameters ensured that this segmentation could be automatically performed on unknown data, with a very low probability to remove suckling events.

\section{Identification of the adequate classifier}

In order to define an appropriate classification model (machine learning model) for associating the suckling status of the calf with the corresponding patterns of different kinematic features, we trained four types of supervised machine learning algorithms - (i) K-Nearest Neighbors (KNN), (ii) Decision tree, (iii) Ensemble classifiers, and (iv) Support Vector Machine (SVM) - using the MATLAB toolbox Statistics and Machine Learning. We repeatedly performed a 60:40 holdout splits on our data (i.e., $60 \%$ of the data as training set and $40 \%$ of the data as testing set) while maintaining class ratios (30 runs): the training set was used for training and the classifier's efficiency was evaluated on the remaining unseen data. The holdout split is a commonly used approach for evaluating general model performance in many machine-learning applications (e.g., Nathan et al., 2012; Carroll et al., 2014; Ladds et al., 2016). Preliminary model selection and hyperparameter tuning for each classifier type were performed using a Bayesian Optimization approach with 5-fold cross-validation. We followed the workflow described in the associated documentation (https://fr.mathworks.com/help/stats/bayesian-optimization-workflow). With the Statistics and Machine Learning toolbox's Bayesian Optimization algorithm, we optimized the different types of supervised machine learning algorithms across a selection of classification models and hyperparameter values in order to pre-select the best classification models that were suitable for our dataset. An optimization run pre-selected one model as the best one by seeking to 
307 minimize classification error. For Ensemble classifiers, the selection of classification models 308 included Bootstrap Aggregation (Bagging), Random Forest (Bag), Random Subspace 309 (Subspace), Adaptive Boosting for Binary Classification (AdaBoostM1), Adaptive Boosting for 310 Multiclass Classification (AdaBoostM2), Gentle Adaptive Boosting (GentleBoost), Adaptive 311 Logistic Regression (LogitBoost), Linear Programming Boosting (LPBoost), Least-Squares 312 Boosting (LSBoost), Robust Boosting (RobustBoost), Random Undersampling Boosting 313 (RUSBoost), and Totally Corrective Boosting (TotalBoost). For KNN classifiers, it included 314 Cityblock, Chebychev, Correlation, Cosine, Euclidean, Hamming, Jaccard, Mahalanobis, 315 Minkowski, Seuclidean, and Spearman metrics-based KNN. For Decision trees, it included 316 classifiers that are based on Gini's diversity index (Gdi), twoing rule, and deviance splitting 317 criterion. For SVM, it included SVM with Gaussian, linear, and polynomial kernels. These 318 different models are detailed in the aforementioned documentation. The number of runs we used for the data splitting (30 runs) and for the Bayesian optimization (100 runs per split per classifier type) were chosen to optimize results stability and processing speed.

For each trial and each class, we calculated five evaluation metrics for making a final decision on the classification model to be retained: Sensitivity (True Positive Rate, Hit Rate or Recall), Precision (Positive Predictive Value), False Positive Rate (FPR), F-score and Global accuracy. These metrics were calculated as follows and used to select the best and most adapted classification model (definitions as in Jeantet et al., 2020):

The Sensitivity measures the ability to detect one behavior among other behaviors:

327 Sensitivity $=\frac{T P}{T P+F N}$

The Precision measures the ability to correctly identify a behavior:

329

330

331

332

333

334

335

336

337

338

339

340

Precision $=\frac{T P}{T P+F P}$

The FPR measures the rate of wrongly considering other behaviors as the behavior of interest. It is related to the Specificity, which is the ability to avoid wrongly considering other behaviors as the behavior of interest:

$$
F P R=1-\text { Specificity } \quad \text { with } \quad \text { Specificity }=\frac{T N}{T N+F P}
$$

The $F$-score measures the accuracy in classifying a behavior. It is the harmonic mean of precision and sensitivity:

$F-$ score $=\frac{2 T P}{2 T P+F P+F N}$

The Global accuracy measures the ability to correctly identify all behaviors as a whole:

Global accuracy $=\frac{T P+T N}{T P+T N+F P+F N}$

These formulas use the following abbreviations: TP - True Positive, TN - True Negative, FN - False Negative), and FP - False Positive. 
To assess the potential influence of training set size reduction on the performance of the elected model, we tested whether changing the training set size changed the performance. We ran a series of trainings with different holdout splitting of the data (training-testing): $60: 40$ as in the original model selection process, 50:50, 40:60, 30:70, 20:80, and 10:90. The process was repeated 30 times for each splitting. A general workflow of the whole machine learning process we followed is presented in Fig. S1.

\section{Generalization across individuals}

To assess whether the model can generalize across different individual whale calves, we also tested a leave-one-out split, similar to Ladds et al. (2016). We trained the model with the highest performance on two calves' data. We then tested its performance on the unseen remaining calf data, establishing if the tag data obtained from individuals could be used to identify the suckling behavior of a different individual. We repeated this analysis three times, leaving out a different calf's data each time following the same machine learning workflow outlined in Fig. S1.

Aside from possible influence of training set size (e.g., the recorded data from the individuals used for training is relatively small), lack of generalization ability across individuals may result from a dataset with high inter-individual variability of suckling behavior characteristics. To address this potential issue, we tested whether excluding features that contribute most to interindividual difference (if any) in the suckling blocks would improve the performance of the supervised machine learning. To identify the features that contribute most to inter-individual difference, we trained a Random Forest algorithm to classify individuals based only on the suckling blocks using the $\mathrm{R}$ software package randomForest (Liaw \& Wiener, 2002). Features that contributed the most to the differentiation by individual were identified as those that had a high Gini index in the Random Forest. We re-ran the leave-one-out design, as described above, but excluding the features identified by using the Gini index. We confirmed the reduction of the inter-individual difference of the suckling blocks by examining the error rate (Out-of-the-bag error rate) of the Random Forest algorithm trained to classify individuals. A reduction of interindividual difference would result in an increase of the error rate. A low error rate indicated that the inter-individual difference is evident enough and thus the Random Forest algorithm is able to make very good classifications.

\section{Results}

\section{Tag deployments}

Four calves were tagged with CATS cam tag in the Sainte Marie channel: one during the calving season of 2018 (identified as Calf1) and three during the calving season of 2019 (identified as Calf2, Calf3, and Calf4). Calf1 and Calf2 were tagged with Cats1 while Calf3 and Calf4 were tagged with Cats2. Their immediate reaction to tagging was a slow evasive swimming, which corresponded to a mild reaction (Stimpert et al., 2012). All of them had unfurled dorsal fins, indicating that they were not neonate calves (Cartwright \& Sullivan, 2009). The deployment on Calf4 was not suitable for our study as the tag (thus the camera) was pointing toward the side of the calf (i.e., away from the mouth) and was thus excluded from our analyses. For Calf3, the data towards the end of the deployment was not usable and thus not analyzed due to lack of visibility on the corresponding video recording as the night approached. Deployment information including date and time is indicated in Table 1. The three deployments provided a total of 10.52 hours of usable video and auxiliary data.

\section{General description of suckling}


385 In all our video recordings, a milk cloud could be observed during or after contact each time the

386

387

388

389

390

391

392

393

394

395

396

397

398

399

400

401

402

403

404

405

406

407

408

409

410

411

412

413

414

415

416

417

418

419

420

421

422

423

424

425

426

427

428

429 calf's snout continuously touched ( $>2 \mathrm{~s}$ ) the mammary slit. All these events appeared to be intentional and were all defined as suckling. We detected 34 suckling events, which lasted on average $18.8 \pm 8.8 \mathrm{~s}$ and represented $1.7 \%$ of the total video deployment (Fig. S2). Detailed summary statistics per individual are presented in Table 1. Most suckling events were clustered in a series of 2-6 suckling events occurring less than a minute apart (Fig. S2). Eighty-eight percent of the suckling events (30 suckling events) were recorded during ten different clusters of suckling events. The global temporal distribution of the suckling events through the duration of each deployment are presented in Fig. S3. There was no evidence of increasing trends in suckling frequency through the duration of the deployments (no indication of a habituation effect, i.e., habituation to tag). Indeed, for the three deployments the first recorded suckling events occurred within the first 90 min of the deployment (within the first 30 min for Calf2) and the periods towards the end of the deployments were not necessarily associated with more frequent suckling events (Fig. S3).

The majority of suckling events occurred while diving, during the descent and the bottom phases ( $N=9$ for descent suckling and $N=20$ for bottom suckling, Table 1). Suckling during descent and during bottom phases of dives occurred at a mean average depth of $16.2 \pm 6.5 \mathrm{~m}$ and $20.2 \pm 6.6 \mathrm{~m}$ respectively (Table 1 ). Only five suckling events occurred at the surface, at a mean average depth of $6.2 \pm 2.7 \mathrm{~m}(\mathrm{~min}$ average $=2 \mathrm{~m}$, max average $=8.4 \mathrm{~m})$. No suckling event occurred during ascent phase of dives.

While calves were visually estimated to be at about $30-45^{\circ}$ pitch angle relative to the midline of their mother's body during suckling in the videos, the pitch recorded by the tag (pitch angle relative to the horizontal) was fairly lower on average (during descent: mean average $=2 \pm 14^{\circ}$, bottom: mean average $=8 \pm 10^{\circ}$, at surface: mean average $=5 \pm 10^{\circ}$, Table 1$)$. Visual estimation of the calf's pitch assumed that the mother had a relatively straight body during nursing, as we observed no apparent extreme body arching of the mother. In all of the observed events, we could visually tell that the calf was always rolling to one side (to the right or to the left). These rolling periods during suckling were recorded by the tag as a sustained deviation of the roll from zero during the event (Fig. S2). In all but one cluster of suckling events, the calf alternated between suckling with a right roll then rotating to suckle with a left roll or vice-versa (Fig. S2). In eleven suckling events (one for Calf1, four for Calf2 and six for Calf3), we were able to visually confirm that the calf's mouth covered only one mammary teat during a suckling event. In three of these events, the tongue was clearly visible and displayed a rhythmic movement (Fig. 1, see also example footage in https:/www.youtube.com/watch?v=nyqhb9BemYI). For these events with confirmed covered teat, rolling to the right corresponded to a mouth covering the right teat $(N=3)$ and rolling to the left corresponded to a mouth covering the left teat $(N=8$, Fig. S2).

\section{Relation between calf's positioning and suckling during dive}

During our tag deployments, Calf1, Calf2, and Calf3 spent respectively $11.7 \%, 12 \%$, and 8.9\% of their total video time in close proximity to their mother's ventral side without suckling. Nonsuckling dives in which the calf remained beneath the mother for at least consecutive $5 \mathrm{~s}$ were more common than suckling dives (Table S1). In other words, using calf proximity to the mother's ventral side alone does not indicate that suckling is occurring. Additional accelerometry singles, which are described below, are thus necessary to determine the occurrence of this behavior.

Peer] reviewing PDF | (2021:10:66429:1:1:NEW 11 Jan 2022) 


\section{Behavioral signatures of suckling}

431 The characteristics of suckling events and non-suckling segments are summarized in Fig. 2. Raw 432 data are provided in Data S1. The detailed results of the LMMs are presented in Table S2 and the 433 detailed results of the post-hoc tests are provided in Table S3. Within our model, for the average depth rate, the effect of suckling, surface phase, and the interaction effect between surface phase and suckling were all statistically non-significant and close to zero (Suckling: $\beta=-0.01, P=$ 0.85 , Surface: $\beta=-0.02, P=0.849$, Suckling*Surface: $\beta=0.03, P=0.863$, Table S2). The effect of descent was on the other hand statistically significant, substantial, and positive $(\beta=0.6, P<$ 0.001 , Table S2). The interaction effect between suckling and descent was also statistically significant and was moderate, although negative ( $\beta=-0.44, P<0.001$, Table S2). In other words, descent phase was associated with a significant increase in average depth rate, which is expected, but the increase is significantly attenuated when suckling. Bottom phase and surface phase were associated to comparable low average depth rate and were not significantly influenced by the act of suckling. There was substantial and statistically significant difference in average depth rate between suckling and non-suckling during descent (Tukey's post-hoc test, $\beta=-0.46, P<0.001$, Table S3). The difference in average depth rate between suckling and non-suckling at the bottom of dive and at surface were however weak and statistically non-significant (Tukey's post-hoc test, Bottom suckling - Bottom non-suckling, $\beta=-0.01, P=1$, Surface suckling - Surface nonsuckling, $\beta=0.01, P=1$, Table S3).

With respect to average speed, the effect of descent phase and surface phase were statistically significant and positive (Descent: $\beta=0.22, P=0.016$, Surface: $\beta=0.3, P=0.033$, Table S2). The effect of suckling, the interaction effect between descent phase and suckling, and the interaction effect between surface phase and suckling were negative but were statistically nonsignificant (Suckling: $\beta=-0.1, P=0.363$, Suckling*Descent: $\beta=-0.21, P=0.22$, Suckling*Surface: $\beta=-0.38, P=0.082$, Table S2). To be specific, descent phase and surface phase were associated with a significant increase in average speed irrespective of the suckling state. Globally, post-hoc tests showed that the average speed tended to be lower during suckling compared to non-suckling for all activity phases (Tukey's post hoc test, negative $\beta$ for all activity phase, Table S3). However, the difference was statistically non-significant (Tukey's post-hoc test, $P>0.05$, Table S3).

Suckling, descent phase and surface phase had moderate and statistically significant positive effect on average Fluke Stroke Rate (FSR) (Suckling: $\beta=0.16, P<0.001$, Descent: $\beta=0.06, P=$ 0.04 , Surface: $\beta=0.1, P=0.016$, Table S2). The interaction of suckling with descent phase and with surface phase had negative but statistically non-significant effect (Suckling*Descent: $\beta=$ $0.06, P=0.258$, Suckling*Surface: $\beta=-0.12, P=0.073$, Table S2). Specifically, descent phase and surface phase were associated with an increase in average FSR and for all activity phases the act of suckling was associated with an increase in average FSR. Post-hoc tests (Table S3) showed however that there was no statistically significant difference in average FSR between suckling and non-sucking except when at bottom (Tukey's post-hoc test, $\beta=0.16, P<0.001$ ).

Regarding the average Overall Dynamic Body Acceleration (ODBA), only descent had statistically significant effect $(\beta=0.16, P<0.028$, Table S2). Suckling and surface phase had statistically non-significant effect (Suckling: $\beta=0.05, P=0.522$, Surface: $\beta=0.1, P=0.377$, Table S2). The interaction of suckling with descent phase and with surface phase also had statistically non-significant effect (Suckling*Descent: $\beta=-0.2, P=0.132$, Suckling*Surface: $\beta=$ $0.1, P=0.551$, Table S2). In other words, descent phase was associated with an increase in average ODBA and for all activity phases, the act of suckling did not significantly influence the 
476

477

478

479

480

481

482

483

484

485

486

487

488

489

490

491

492

493

494

495

496

497

498

499

500

501

502

503

504

505

506

507

508

509

510

511

512

513

514

515

516

517

518

519

520

521

average ODBA. Overall, there was no statistically significant difference in average ODBA between suckling and non-sucking for all activity phases (Tukey's post-hoc test, $-0.15 \leq \beta \leq 0.05$, $P>0.05$, Table S3).

For average pitch, the effect of surface phase and the interaction effect between surface phase and suckling were weak and statistically non-significant (Surface: $\beta=-5.05, P=0.222$, Suckling*Surface: $\beta=1.41, P=0.829$, Table S2). The effect of suckling was weak, yet statistically significant $(\beta=6.36, P=0.044$, Table S2). The effect of descent phase was on the other hand statistically significant, substantial, and negative $(\beta=-18.26, P<0.001$, Table S2). The interaction effect of suckling on descent phase was also statistically significant and moderate but positive $(\beta=12.77, P=0.011$, Table $\mathrm{S} 2)$. Specifically, descent phase was associated with a significant decrease in average pitch, which is expected, but the decrease was significantly attenuated when suckling (posture close to the horizontal when suckling). Bottom phase and surface phase were associated to an average pitch close to zero, positively influenced by the act of suckling (slight upward pitch when suckling). There was substantial and statistically significant difference of average pitch between suckling and non-suckling during descent (Tukey's post-hoc test, $\beta=19.13, P<0.001$, Table S3). The difference in average pitch between suckling and non-suckling at the bottom of dive and at surface were however weak and statistically non-significant (Tukey's post-hoc test, Bottom suckling - Bottom non-suckling, $\beta=$ $6.36, P=0.345$, Surface suckling - Surface non-suckling, $\beta=7.77, P=0.756$, Table S3).

The effect of suckling on average absolute roll was statistically significant and very strong ( $\beta$ $=38.46, P<0.001$, Table S2). All other effects were statistically non-significant and mostly weak (Descent: $\beta=-0.44, P=0.882$, Surface: $\beta=0.64, P=0.885$, Suckling*Descent: $\beta=-0.25$, $P=0.965$, Suckling*Surface: $\beta=-11.56, P=0.108$, Table S2). In other words, all activity phases were associated with fairly low average absolute roll and the average absolute roll was positively influenced by suckling, regardless of the activity phase, i.e., the average absolute roll was high during suckling for all activity phase. Post-hoc test indicated a significant difference in average absolute roll between suckling and non-sucking for all activity phases (Tukey's post-hoc test: $26.89 \leq \beta \leq 38.46, P \leq 0.001$, Table S3).

The effect of suckling, descent phase and surface phase, and the interaction effect between descent phase and suckling and between surface phase and suckling on the average roll rate were all low and statistically non-significant (Suckling: $\beta=1.17, P=0.06$, Descent: $\beta=0.32, P=$ 0.559 , Surface: $\beta=0.28, P=0.726$, Suckling*Descent: $\beta=0.4, P=0.692$, Suckling*Surface: $\beta$ $=1.51, P=0.249$, Table S2). The average roll rate displayed by calf during bottom phase, descent phase, and surface phase were comparable and the average roll rate was not significantly influenced by suckling. Overall, there was no statistically significant difference of roll rate between suckling and non-sucking for all activity phases (Tukey's post-hoc test: $\beta \leq 2.68, P>$ 0.05 , Table S3).

\section{Best classifier for automatic identification of suckling}

A total of 7827 behavioral blocks of $2 \mathrm{~s}$ duration (4697 for training and 3130 for testing, 60:40 holdout splitting) were obtained from the datasets after removing blocks too close to the surface and those of high speed activities (see Segmentation in the Method section). The raw data used for this part of the study are available in Data S2. No suckling events were affected by our thresholds for removing blocks too close to the surface and those of high speed activities. The class 'suckling' represented $4 \%$ of the data and the class 'non-suckling' represented $96 \%$. Fig. $3 \mathrm{~A}$ presents all models pre-selected by the optimization procedure for identifying automatically suckling and non-suckling in non-labelled data. How often each model was selected over the 30 
522 runs of the optimization procedure for each type of classifier is presented in Fig. 3A, and details 523 are presented in Table S4.

524

525

526

527

528

529

530

531

532

533

534

535

536

537

538

539

540

541

542

543

544

545

546

547

548

549

550

551

552

553

554

555

556

557

558

559

560

561

562

563

564

565

566

567

All pre-selected classification models had a mean global accuracy $>96.93 \%$ on unseen data (Table S4). They all identified suckling events with a low False Positive Rate (mean FPR < 1\%) except for SVM with polynomial kernels (mean FPR > 1\% but not exceeding 2\%, Table S4). The likelihood of misclassifying an event as suckling when it should have been classified as nonsuckling is thus extremely low for most of the pre-selected models. As shown in Fig. 3A, the SVM with linear kernel model and all decision trees are more located at the bottom left quadrant of the figure, indicating that they had a lower Precision and Sensitivity with regard to suckling, in contrast to AdaBoostM1, GentleBoost, Minkovski metrics-based KNN, Cityblock metricsbased KNN, and Cosine metrics-based KNN models that lay on the top right of the figure. The SVM with polynomial kernel model lays on the top left of the figure, indicating that it had a high Sensitivity but had a low Precision. On the other hand, the Bag or Random Forest model, the SVM with Gaussian kernel model, and the Cosine metrics-based KNN model lay on the bottom right, indicating that they had high Precision but had low Sensitivity. The AdaBoostM1, an Ensemble classifier, was the model presenting the highest $F$-score. It had the highest Sensitivity while having at the same time a very good Precision (red circle symbol in Fig. 3A). It was therefore the best model for correctly identifying suckling events. Examples of classification resulting from the AdaBoostM1 are presented in Fig. 3B and Fig. 3C. In these examples, the AdaboostM1 model only misclassified non-suckling blocks as suckling in very rare cases (False Positives, 8 in Fig. 3B and null in Fig. 3C - no red cross). However, it misclassified suckling blocks as non-suckling more often (False Negatives, 34 in Fig. $3 \mathrm{~B}$ and 6 in Fig. 3C - blue crosses). Nevertheless, it detected most of the real suckling blocks (True Positives, 90 in Fig. 3B and 15 in Fig. 3C - red circles).

As shown in Fig. 3D, while there was typical decrease in the performance of the AdaBoostM1 when we reduced the training set size, the model still detected substantial amount of suckling blocks and had a relatively good precision even when using only $10 \%$ of the data as training set (mean at 10:90 split for suckling: sensitivity $=0.49 \pm 0.08$, precision $=0.79 \pm 0.06, F$-score $=$ $0.60 \pm 0.06, N=30$ ). However, the results seemed more variable (high SD) when training set was really small (Fig. 3D).

\section{Generalization of the supervised machine learning across individuals}

We evaluated the generalization ability of the AdaBoostM1 model, the best identified classifier in the context of suckling identification, using a leave-one-out design. The goal was to assess whether we can use data from other individuals to detect the suckling behavior of a new individual. Given that we had three individuals, there were only three possible splitting combinations: (1) blocks from Calf2 and Calf3 as training set (5766 blocks, 4.5\% suckling and 95.5\% non-suckling) and blocks from Calf1 as testing set (2061 blocks, 2.6\% suckling and 97.4\% non-suckling), (2) blocks from Calf1 and Calf3 as training set (7256 blocks, 3.6\% suckling and $96.4 \%$ non-suckling) and blocks from Calf2 as testing set (571 blocks, 8.6\% suckling and 91.4\% non-suckling), and (3) blocks from Calf1 and Calf2 as training set (2632 blocks, 3.9\% suckling and 96.1\% non-suckling) and blocks from Calf3 as testing set (5195 blocks, 4\% suckling and 96\% non-suckling).

Results of the leave-one-out design are presented in Fig. 4. All combinations were more located at the bottom part of the Sensitivity versus Precision plot (open symbols). This indicated that the quantity of the detected suckling blocks was low (low Sensitivity) regardless of the combination. The quality was however still good (relatively high Precision) for the first and

Peer) reviewing PDF | (2021:10:66429:1:1:NEW 11 Jan 2022) 
568 second combinations (open square and open diamond symbols). The third combination (open 569 triangle symbol) was on the other hand more located at the left of the plot, indicating that the 570 quality of the classification was bad (low Precision). Globally, it can be said that while some

571

572

573

574

575

576

577

578

579

580

581

582

583

584

585

586

587

588

589

590

591

592

593

594

595

596

597

598

599

600

601

602

603

604

\section{Discussion}

606 Our study characterized the suckling behavior in humpback whale calves less than 3-month old

607

608

609

610

611 good quality classification can be obtained, it still depended on the combination used.

As inter-individual variation of the suckling blocks' kinematic features may have influenced the generalization ability of the AdaBoostM1, we assessed the model's performance when the features that contributed the most to inter-individual difference were excluded. We performed Random Forest classification of the individuals to check the existence of inter-individual differences on suckling blocks, analyzed the features' contribution to the differentiation, and identified a set of features to be excluded in order to reduce the inter-individual difference. The Random Forest algorithm indicated that there was effectively a strong inter-individual difference. Indeed, the classification error rate, indicated by the Out-of-the-bag error rate, was very low (Out-of-the-bag error rate $=3.22 \%$ ). As shown in the Fig. 5A, the Random Forest algorithm was able to correctly predict to which individual each suckling blocks belongs to in most of the cases (correspondence between true class and predicted class). The Fig. 5B shows the Gini index of each feature. A high Gini index indicated that the feature played great role in the classification (in other words, in the individual differentiation). In the plot, we noticed a sharp drop in importance after the $9^{\text {th }}$ feature. We thus chose to exclude the first 9 features (Gini index $>4$ ): the maximum depth, mean depth, maximum pitch, minimum pitch, mean pitch, minimum depth, mean speed, minimum speed, and the maximum speed. The Fig. $5 \mathrm{C}$ shows the new resulting classification of the suckling blocks by individuals when these features were not included. The classification error rate increased notably (Out-of-the-bag error rate $=22.19 \%$ ). The Random Forest algorithm confused the individual attribution of the suckling blocks more often (more non-corresponding true class and predicted class), indicating that we successfully reduced partially the inter-individual differences. We observed a substantial increase in performance when we re-ran the leave-one-out design using the restricted list of features (mean FSR, mean absolute roll, minimum absolute roll, minimum depth rate, maximum absolute roll, depth rate variance, maximum ODBA, mean ODBA, mean depth rate, ODBA variance, pitch variance, maximum depth rate, speed variance, minimum ODBA, maximum roll rate, absolute roll variance, depth variance, mean roll rate, depth skewness, ODBA skewness, minimum roll rate, ODBA kurtosis, absolute roll kurtosis, roll rate kurtosis, speed kurtosis, absolute roll skewness, depth rate skewness, speed skewness, pitch skewness, roll rate skewness, pitch kurtosis, depth rate kurtosis, roll rate variance, depth kurtosis) (Fig. 4, blue symbols). Indeed, the Global accuracy was higher than when including all features, and the FPR for suckling blocks decreased. Most importantly, the quantity of detected suckling blocks increased (higher Sensitivity). Also the quality of the classification improved globally (increased Precision), even for the combination that had a very poor Precision at first. using CATS cam tags on one calving ground in the South Western Indian Ocean. Several important aspects of this vital behavior were investigated: (i) duration and frequency, (ii) occurrence in the water column, (iii) modalities, (iv) behavioral signatures, and (v) the possibility to use only acceleration and depth-derived data (i.e. depth, depth rate, speed, Fluke Stroke Rate (FSR), Overall Dynamic Body Acceleration (ODBA), pitch, roll, roll rate, which were obtained 
612 using the data from the depth sensor and/or the 3-axis accelerometer only) to perform an

613 automatic detection of suckling events.

\section{Advantage of using animal-borne camera-equipped multi-sensor tags}

615 Animal-borne multi-sensor tags equipped with video camera are very efficient for studying

616 suckling behavior since they can deliver a view from calf's perspective and thus confirm

617 evidence of suckling. Video from tags allowed differentiation of events in which the calf started

618 to intentionally touch its mother's mammary slit to suckle from events when the calf was only

619 positioning itself under the mother. Such differentiation between suckling and non-suckling

620 periods would not be possible if the visual observations were performed from a different

621 perspective or at distance (e.g., surface ship-based, or aerial drone-based), therefore leading to

622 biased estimations of suckling behavior.

623 In our study, the view offered by the video camera allowed clear definition of suckling events

624 initiated by physical contact between the calf's snout and the mammary teat. Such contact did

625 not happen every time the calf was under the mother. Suckling behavior was further confirmed

626 by the presence of a milk cloud as in Tackaberry et al. (2020). This method greatly improved

627 describing suckling behavior with confidence.

628 Previously, positioning and posture of the calves have been used frequently as a proxy for 629 determining the occurrence of nursing or suckling in humpback whales (Glockner \& Venus, 630 1983; Glockner-Ferrari \& Ferrari, 1985; Clapham \& Mayo, 1987; Morete et al., 2003; Videsen et 631 al., 2017; Zoidis \& Lomac-MacNair, 2017). As previously shown by Tackaberry et al. (2020) with older calves (studied in their feeding grounds), we found that young calves were also positioned frequently under the mother and in close proximity but they were actually not suckling. Furthermore, suckling dives (i.e., dives during which suckling was visually confirmed by evidence of a milk cloud) appeared to be much rarer in comparison to non-suckling dives during which the calf was observed under the mother. Consequently, great care must be taken when associating calf positioning with suckling or with suckling dives as these are not necessarily associated. The calf, when positioning under the mother while not necessarily suckling may be taking advantage of reduced drag, as shown for dolphin calves (Noren \&

640 Edwards, 2011).

\section{Duration and frequency of suckling events}

642 Suckling events were brief and rare $(18.8 \pm 8.8 \mathrm{~s}$ on average and $<2 \%$ of observation time). They

643 were shorter in duration compared to those observed by divers in another breeding area $(30.6 \pm 17$

644 s on average, Zoidis \& Lomac-MacNair, 2017). This difference might be related to the method

645 used, as discussed above. The average suckling duration reported in the feeding area is quite

646 similar to what we observed (23 $\pm 7 \mathrm{~s}$, Tackaberry et al., 2020). The associated suckling frequency

647 was however much lower in the feeding area $(0.3 \%$ of the time for an observation between 0745

648 and 1700 hours, Tackaberry et al., 2020) compared to what we observed in the calving area

649 (1.7\% between 1000 and 1800 hours). This expected difference suggests suckling rate varies

650 with age (greater for young calves in the breeding grounds versus older calves in the feeding

651 grounds), as is the case with several species of pinnipeds (Oftedal, Boness \& Tedman, 1987).

\section{Occurrences of suckling in the water column}

653 As in Tackaberry et al. (2020), most of the suckling events occurred at depth and during dives.

654 Humpback whales may favor nursing at depth to help the calf in maintaining the suckling

655 posture as the latter would be more buoyant at surface, or for facilitating the thermoregulation of 
656 the adult female (Videsen et al., 2017). We noticed that the rare surface suckling events only

657 occurred at the end of the day, when the sun was less intense, suggesting a possible link between

658

659

660

661

662

663

664

665

666

667

668

669

670

671

672

673

674

675

676

677

678

679

680

681

682

683

684

685

686

687

688

689

690

691

692

693

694

695

696

697

698

699

700

701

surface nursing events and thermoregulation behavior. Further investigations on suckling during the night are needed for comparison, as most videos were recorded during daylight only (this study; Tackaberry et al., 2020). Nursing behavior might show different patterns at night for the reasons cited above. If indeed suckling at night is more common and is occurring more at the surface, this has implications for the risk of boat-strikes at a time when visual cues are limited (for those at the helm).

The maximum suckling depth we recorded $(32.6 \mathrm{~m})$ was much shallower than the maximum reported by Tackabery et al. (2020). However, this does not necessarily relate to any physical limits of young calves. Rather, this maximum suckling depth might be an effect of the environment. Indeed, the average depth of the Sainte Marie channel is $35 \mathrm{~m}$ (Trudelle et al., 2016) and in our video recordings it was common to see the seabed when the whales dove to around $30 \mathrm{~m}$ depth.

All suckling events during dives occurred during the descent and bottom phases only, unlike in Tackaberry et al. (2020) where they also observed suckling events during the ascent phase. One possible explanation is that very young calves found in breeding grounds have less breath capacities (Saloma, 2018) than older calves found in feeding areas. Thus, during the ascent phase of their dive, their priority would be to reach the surface to breathe rather than to suckle.

\section{Suckling modalities}

With respect to posture, as estimated from the video recordings, the calf positioned itself at 30$45^{\circ}$ pitch angle relative to the midline of the mother's body when suckling. This is in agreement with past observations (Glockner-Ferrari \& Ferrari, 1985; Zoidis \& Lomac-MacNair, 2017). However, the pitch recorded by the tags (pitch angle relative to the horizontal) during suckling was always relatively low on average $\left(<15^{\circ}\right)$. This suggests that the mother is generally oriented facing slightly downward when nursing its calf even during phases other than the descent (during which the mother does not otherwise have to lean downward).

One of the most innovative findings of our study was the calf's tendency to roll continuously to the side when suckling. Our data confirm the correspondence between the rolling side and the suckled teat in nine events. The calves rolled to the left to suckle on the left teat and rolled to the right to suckle on the right teat. The rolling behavior might be thus related to the anatomy of the mammary gland, in particular the orientation of the nipple as the nipple is extruded from the mammary slit. If each breast is positioned so that the teat is directed at an angle relative to the midsagittal plane, then the calf would be forced to match that angle with its mouth in order to stimulate the nipple to extrude and then grasp the nipple with its tongue. Video recordings showed the calf's tongue is directed laterally, visible in the oral gape of the mouth on one side of the head. The calf must roll to one side in order to align the lateral aspect of its mouth with the mammary slit (rolling left to align its right oral gape against the mother's left breast, and vice versa).

There was also the clear pattern of alternation from one side to the other between successive suckling events. Why then must the calf alternate sides? Perhaps the milk supply of each breast is limited, forcing the calf to suckle both sides in order to obtain a sufficient volume for satiation. This would ensure that the mother's breasts continue to lactate evenly on both sides. Another reason may be related to the milk production/storage/ejection system. It is widely accepted that milk is voluntarily ejected by the mother into the calf's mouth in cetaceans (Slijper, 1966). It is possible that the amount of milk the mother can continuously eject is limited and the gland may

Peer] reviewing PDF | (2021:10:66429:1:1:NEW 11 Jan 2022) 
702 need a refractory period during which it must reset before the next ejection can occur. If so, then

703 alternating which side to suckle maximizes feeding for the calf while increasing milk delivery

704 efficiency for the mother. In this scenario, the currently suckled breast performs milk ejection

705 while the previously suckled breast refills in preparation for the next ejection. This would thus

706 force the calf to alternate between the two mammary glands to get enough milk during the

707 successive nursing bouts. In-depth anatomical investigation is needed to answer this question, as

708 there is scant literature on whale lactation or breast anatomy. The pattern of alternation between

709 the two mammary gland, if indeed necessary and related to the milk production/storage/ejection

710 system in whales, may have an implication on the post-partum viability of twins in cetaceans.

711 Regarding the anatomy of suckling, it is important to note that in a few cases, rhythmic

712 movements of the calf's tongue were observed during suckling events. This suggests that the calf

713 actively participates in directing milk into its mouth. It is unclear whether these movements are

714 stimulatory to the mother's 'let down' reflex (nipple erection and milk ejection), serve to 'strip'

715 the nipple (as occurs in many land mammals to squeeze milk out), or create a piston-like suction

716 to draw out milk (perhaps in combination with milk ejection from the mother). The lateral aspect

717 of the calf's rostral tongue is comprised of elongated marginal papillae that may serve to grasp

718 the nipple. This latching-on-nipple function has been proposed for newborn tongues in other

719 whale species (Kastelein \& Dubbeldam, 1990; Shindo et al., 2008; Ferrando et al., 2010; Kienle

720 et al., 2015). These marginal papillae may also "zipper" together to form a tube for channeling

721 milk into the mouth, compensating for the calf's lack of lips and cheeks to seal and contain the

722 nipple and the extruded milk. Alternatively, the rhythmic movements of the tongue may be only

723 an artefact produced by the calf's swimming movements as the calf undulates its body to

724 maintain its suckling position while the tongue maintains constant contact with the nipple.

725 Further study of the calf tongue may provide additional insights to its function.

726

727

728

729

730

731

732

733

734

735

736

737

738

739

740

741

742

743

744

745

\section{Behavioral signatures of suckling events}

The comparison of the data derived from accelerometer and depth sensor for suckling and nonsuckling events revealed mainly one distinctive characteristic of the suckling behavior: a high absolute roll. Other characteristics, although not obvious, such as high FSR and low speed are also suggested by the results of our comparisons. For suckling occurring during the descent phase, there were additional notable specificities: a low depth rate and a pitch close to zero. This is in sharp contrast with descent non-suckling where calves showed a high descent rate on average and were directed downwards.

To our knowledge, no study to date has highlighted the characteristics of suckling behavior of whales in terms of roll recorded by an accelerometer. The sustained roll deviance from zero during suckling events is directly related to the aforementioned rolling pattern observed in the videos: rolling to the side may facilitates access to a particular mammary slit.

With respect to the kinematics, lower speed while still deploying some physical effort (high FSR) when suckling is consistent with the results of Tackaberry et al. (2020). Even though the pair makes little or no forward movement during suckling, the calf still has to maintain actively the suckling posture to stay in physical contact with the mammary slit. Unlike Tackabery et al. (2020), we however did not found any specificity of suckling with respect to ODBA. Concerning the descent suckling, the relatively low depth rate may help the calf in maintaining the suckling posture.

\section{Automatic detection of suckling using accelerometer and depth-derived data}


746 In the field of ethology, the advances in machine learning have offered the opportunity to 747 classify behaviors within a complex database (Valletta et al., 2017). Given the stereotyped traits

748 of suckling behavior in humpback whale calves, we tested whether it was possible to apply

749 supervised machine learning algorithms on labelled accelerometer and depth sensor data to

750

751

752

753

754

755

756

757

758

759

760

761

762

763

764

765

766

767

768

769

770

771

772

773

774

775

776

777

778

779

780

781

782

783

784

785

786

787

788

789

790

791 automatically discern suckling from non-suckling periods. Although accelerometer data have been already largely coupled with supervised machine learning to detect behaviors in various species (Nathan et al., 2012; Carroll et al., 2014; Ladds et al., 2016; Jeantet et al., 2020), our study is the first attempt to use it for humpback whales and in the framework of a suckling behavior study.

The biggest challenge in the automatic classification of suckling behavior is probably the class imbalance. Indeed, suckling events are naturally rare (this study; Tackaberry et al., 2020). It is argued that unbalanced data tend to bias predictions in favor of the majority class. This bias is problematic in situations in which missing the minority class case is worse than misclassifying a majority class (Leevy et al., 2018). In the case of suckling versus non-suckling classification, this bias is not a significant concern since there is more interest in minimizing the False Positive Rate (FPR) for the targeted behavior (i.e. suckling). Such conservative measure is generally adopted in behavioral detection (Nathan et al., 2012; Carroll et al., 2014; Tennessen et al., 2019). On the other hand, the best model we found for identifying suckling events, the AdaBoostM1, is a model suitable for unbalanced binary classifications (Galar et al., 2011; Leevy et al., 2018). Thus, class imbalance is less of a concern. In order to avoid evaluation bias, the evaluation process was guided primarily by the minimization of the FPR rather than the Global accuracy, since the latter does not distinguish between the numbers of correctly classified examples of different classes and can be misleading in the framework of an unbalanced dataset (Galar et al., 2011). It is important to recall that we also performed a data segmentation (we excluded blocks at $<1.5 \mathrm{~m}$ depth or at $>2 \mathrm{~m} \mathrm{~s}^{-1}$ speed) that helped in reducing data imbalance and in improving the results. While the thresholds we chose for the segmentation works well with our data and are compatible with our current knowledge on the suckling behavior of humpback whale, they may be subject to change and adjustment as our knowledge on humpback whale behavior evolves.

With an initial 60:40 holdout training-testing split, the average Sensitivity and Precision of the AdaBoostM1 model with respect to suckling class were of 0.71 and 0.89 respectively and the mean FPR was $<1 \%$. In other words, the model was able to detect the vast majority of suckling blocks and about 9 out of 10 blocks reported as suckling corresponded to visually confirmed suckling periods.

The Leave-one-out design (blocks from two individuals as training set and the one remaining individual as a testing set) allowed testing whether data from two individuals can be used to detect suckling behavior in a novel individual. In this design, it was expected that the performance would drop notably (lower Sensitivity and Precision). Indeed, the inter-individual difference has been shown to generally penalize the performance of automatic classification models (Vázquez Diosdado et al., 2015; Ladds et al., 2016). When we reduced the interindividual difference in terms of suckling by excluding features that introduce substantial interindividual differences, the performance returned to a reasonable level regardless of the combination (i.e., the model generalized better and became more robust). Indeed, out of every 10 blocks reported as suckling, at least about 6 blocks were confirmed to be correct (lowest Precision $=0.59$ ). The associated Sensitivity was relatively low, indicating that several suckling blocks were not detected. However, detecting only few blocks but with precision is acceptable in the case where the goal is to be sure that a positive result means the identified suckling blocks

Peer) reviewing PDF | (2021:10:66429:1:1:NEW 11 Jan 2022) 
792 really correspond to suckling periods. Furthermore, with only few blocks detected with high

793 confidence, complete suckling events (start and end) may be determined by examining the

794 corresponding sustained roll deviations, thus compensating the lack of sensitivity.

795 We were not able to investigate the effect of individual tag differences due to limited sample.

796 We can however expect that this effect, if any, is relatively small. In the first and second

797 combinations of our leave-one-out experiments, the two calves used for training were tagged

798 with different individual tags (Cats 1 and Cats2) and the remaining calf used for testing was

799 tagged with one of the individual tags involved in training (Cats1 or Calf2). However, in the

800 third combination, the two calves used for training were tagged with the same individual tag

801 (Cats1) and the remaining calf used for testing was tagged with a different individual tag (Cats2).

802 The fact that there was no evident divergence of the results of our third combination from the

803 results of the first and second combinations suggests that our model was not significantly

804 affected by the differences in individual tags. Further studies are encouraged regarding the

805 potential effect of individual tag differences since our conclusion is still limited by sample size.

806

807

808

809

810

811

812

813

814

815

816

817

818

819

820

821

822

823

824

825

826

827

828

829

830

831

832

833

834

835

As more data is added to the learning set, we can expect better performance from the models. Indeed, we showed that the size of the training set can slightly influence the accuracy of the results. Similarly, we can also expect that with more individuals, the constraints and restrictions related to inter-individual differences would also be reduced (Ladds et al., 2016).

These results on automatic identification of suckling events are very promising for rapid evaluation of larger data sets and will open new opportunities for investigating limited data sets. First, the model may be adjusted to accommodate for incomplete observations (altered $/ \mathrm{missing}$ video file, night-time recording, etc.) using only two sensors (3-axis accelerometer and depth sensor). Secondly, the model may be applied to data from comparable multi-sensor tags that lack visual support (video recordings lacking or too dark, tags without camera such as Acousonde and Dtags, etc.).

While these methods will broaden the opportunities in the domain of humpback whale mother-calf behavioral studies (and eventually may be applied to studying other large whales), care must be taken in these early stages. Until the acquisition of large training data occurs (several individuals), researchers should be aware of the limitations of each trained model. For example, possible differences in gaits and behavioral pattern with respect to age (Noren, Biedenbach \& Edwards, 2006; Saloma, 2018) may hinder the generalization ability of the models. For our trained model, we caution that it should be conservatively applied to less than 3month old non-neonate calves occurring in a shallow water. Nevertheless, a concrete application example of the automatic identification of suckling behavior would be the investigation of the behavioral time budget of mother-calf pairs over 24 hours (day and night). The main difficulty in using multi-sensor tags for studying the behaviors of mother-calf pairs concerns the acquisition of sufficiently representative data. Indeed, tagging whales is a challenge per se and some age classes are more difficult to observe (e.g., neonate calves are rarely observed). Moreover, the tag attachment time on a whale is often limited (few hours on average), thus it is rare to record behaviors of individual whales over long periods ( $>24$ hours). Future studies should address the ontogeny aspect of calves' behavior in order to subsequently assess its implication in the field of automatic behavior detections. The development of more durable yet non-invasive tag attachment systems is also highly encouraged as this is the key to acquiring more complete data and thus obtaining more representative learning set for accurate automatic behavior detection.

\section{Conclusions}


837 Our results provide new knowledge on the suckling behavior of humpback whale calves in their 838 calving ground. Our descriptions, based on videos from the calf's perspective, accelerometer 839 data, and depth data complement previous studies based on surface and sub-surface observations 840 (Glockner \& Venus, 1983; Glockner-Ferrari \& Ferrari, 1985; Clapham \& Mayo, 1987; Morete et 841 al., 2003; Videsen et al., 2017; Zoidis \& Lomac-MacNair, 2017). We found that suckling is more 842 frequent compared to what has been reported in the feeding area (Tackaberry et al., 2020), 843 suggesting a variation of the suckling rate with the calf's age and underlining the importance of 844 the suckling behavior in the calving area. We also confirmed that most suckling events occur at 845 depth, highlighting the importance of using multi-sensor tags equipped with a video camera in 846 order obtain reliable observations. Finally, we found that suckling behavior is stereotyped, 847 especially characterized by a continuously sustained roll deviating significantly from zero and 848 likely a high FSR and low speed, and can potentially be detected automatically by supervised machine learning. While our results are only based on three tag deployments on humpback whale calves in the breeding ground, they are a proof of method on which future investigations of suckling and nursing behavior in humpback whales can build upon. Our study should be replicated with other baleen whale species for which suckling behavior is still undiscovered.

853

854

855

856

857

858

859

860

861

862

863

864

865

866

867

868

869

870

871

872

873

874

875

876

877

878

879

880

\section{Acknowledgements}

We would like to warmly thank Aina F. Ramanampamonjy, Léo Duperret, Mandrindra O. Rakotovao and, Nyal Mueenuddin who contributed to the data collection. We warmly thank Conor Ryan, Jennifer Tackaberry, and one anonymous referee for their valuable and constructive comments on the manuscript.

\section{References}

Balshine S. 2012. Patterns of parental care in vertebrates. Oxford University Press Oxford. DOI: 10.1093/acprof:oso/9780199692576.001.0001.

Bannister JL. 2018. Baleen whales (Mysticeti). In: Encyclopedia of Marine Mammals. Elsevier, 62-69. DOI: 10.1016/B978-0-12-804327-1.00058-3.

Baraff L, Weinrich MT. 1993. Separation of humpback whale mothers and calves on a feeding ground in early autumn. Marine Mammal Science 9:431-434. DOI: 10.1111/j.17487692.1993.tb00476.x.

Braithwaite JE, Meeuwig JJ, Hipsey MR. 2015. Optimal migration energetics of humpback whales and the implications of disturbance. Conservation Physiology 3:cov001. DOI: 10.1093/conphys/cov001.

Butcher P, Colefax A, Gorkin R, Kajiura S, López N, Mourier J, Purcell C, Skomal G, Tucker J, Walsh A, Williamson J, Raoult V. 2021. The Drone Revolution of Shark Science: A Review. Drones 5:8. DOI: 10.3390/drones5010008.

Cade DE, Barr KR, Calambokidis J, Friedlaender AS, Goldbogen JA. 2017. Determining forward speed from accelerometer jiggle in aquatic environments. Journal of Experimental Biology 221:jeb170449. DOI: 10.1242/jeb.170449.

Cade DE, Friedlaender AS, Calambokidis J, Goldbogen JA. 2016. Kinematic Diversity in Rorqual Whale Feeding Mechanisms. Current Biology 26:2617-2624. DOI: 10.1016/j.cub.2016.07.037.

Carroll G, Slip D, Jonsen I, Harcourt R. 2014. Supervised accelerometry analysis can identify prey capture by penguins at sea. Journal of Experimental Biology 217:4295-4302. DOI: 10.1242/jeb.113076. 
881 Cartwright R, Sullivan M. 2009. Behavioral ontogeny in humpback whale (Megaptera

882 novaeangliae) calves during their residence in Hawaiian waters. Marine Mammal Science

883

884 25:659-680. DOI: 10.1111/j.1748-7692.2009.00286.x.

Chittleborough RG. 1958. The breeding cycle of the female humpback whale, Megaptera nodosa (Bonnaterre). Marine and Freshwater Research 9:1-18. DOI: 10.1071/MF9580001.

885

Clapham PJ. 2018. Humpback Whale. In: Encyclopedia of Marine Mammals. Elsevier, 489-492. DOI: 10.1016/B978-0-12-804327-1.00154-0.

887

888

889

890

891

892

893

894

895

896

897

898

899

900

901

902

903

904

905

906

907

908

909

910

911

912

913

914

915

916

917

918

919

920

921

922

923

924

925

926

Clapham PJ, Mayo CA. 1987. Reproduction and recruitment of individually identified humpback whales, Megaptera novaeangliae, observed in Massachusetts Bay, 1979-1985. Canadian Journal of Zoology 65:2853-2863. DOI: 10.1139/z87-434.

Corkeron PJ, Connor RC. 1999. Why do baleen whales migrate? 1. Marine Mammal Science 15:1228-1245. DOI: 10.1111/j.1748-7692.1999.tb00887.x.

Daly M. 1979. Why don't male mammals lactate? Journal of Theoretical Biology 78:325-345. DOI: 10.1016/0022-5193(79)90334-5.

Faria M-A, DeWeerdt J, Pace F, Mayer F-X. 2013. Observation of a humpback whale (Megaptera novaeangliae) birth in the coastal waters of Sainte Marie Island, Madagascar. Aquatic Mammals 39:296. DOI: 10.1578/AM.39.3.2013.296.

Ferrando T, Caresano F, Ferrando S, Gallus L, Wurtz M, Tagliafierro G. 2010. The tongue morphology and lingual gland histochemistry of Ligurian Sea odontocetes. Marine mammal science 26:588-601.

Friard O, Gamba M. 2016. BORIS: a free, versatile open-source event-logging software for video/audio coding and live observations. Methods in ecology and evolution 7:1325-1330. DOI: 10.1111/2041-210X.12584.

Galar M, Fernandez A, Barrenechea E, Bustince H, Herrera F. 2011. A review on ensembles for the class imbalance problem: bagging-, boosting-, and hybrid-based approaches. IEEE Transactions on Systems, Man, and Cybernetics, Part C (Applications and Reviews) 42:463-484. DOI: 10.1109/TSMCC.2011.2161285.

Gittleman JL, Thompson SD. 1988. Energy allocation in mammalian reproduction. American zoologist 28:863-875. DOI: 10.1093/icb/28.3.863.

Glockner-Ferrari DA, Ferrari MJ. 1985. Individual identification, behavior, reproduction, and distribution of humpback whales, Megaptera novaeangliae, in Hawaii. US Marine Mammal Commission.

Glockner DA, Venus SC. 1983. Identification, growth rate, and behaviour of humpback whale (Megaptera novaeangliae) cows and calves in the waters off Maui, Hawaii, 1977--79: 223-258 in Payne, R.(ed), Communication and behavior of whales.

Hall WG, Hudson R, Brake SC. 1988. Terminology for use in investigations of nursing and suckling. Developmental psychobiology 23:89-91. DOI: 10.1002/dev.420210107.

Iwata T, Biuw M, Aoki K, Miller PJO, Sato K. 2021. Using an omnidirectional video logger to observe the underwater life of marine animals: Humpback whale resting behaviour. Behavioural processes 186:104369. DOI: 10.1016/j.beproc.2021.104369.

Jeantet L, Planas-Bielsa V, Benhamou S, Geiger S, Martin J, Siegwalt F, Lelong P, Gresser J, Etienne D, Hiélard G, others. 2020. Behavioural inference from signal processing using animal-borne multi-sensor loggers: a novel solution to extend the knowledge of sea turtle ecology. Royal Society open science 7:200139. DOI: 10.1098/rsos.200139.

Johnson MP, Tyack PL. 2003. A digital acoustic recording tag for measuring the response of wild marine mammals to sound. IEEE journal of oceanic engineering 28:3-12. DOI:

Peer) reviewing PDF | (2021:10:66429:1:1:NEW 11 Jan 2022) 
927

928

929

930

931

932

933

934

935

936

937

938

939

940

941

942

943

944

945

946

947

948

949

950

951

952

953

954

955

956

957

958

959

960

961

962

963

964

965

966

967

968

969

970

971

972

10.1109/JOE.2002.808212.

Kastelein RA, Dubbeldam JL. 1990. Marginal papillae on the tongue of the harbour porpoise (Phocoena phocoena), bottlenose dolphin (Tursiops truncatus) and Commerson's dolphin (Cephalorhynchus commersonii). Aquatic Mammals 15:158-170.

Kienle SS, Ekdale EG, Reidenberg JS, Deméré TA. 2015. Tongue and hyoid musculature and functional morphology of a neonate gray whale (Cetacea, Mysticeti, Eschrichtius robustus). The Anatomical Record 298:660-674. DOI: 10.1002/ar.23107.

Ladds MA, Thompson AP, Slip DJ, Hocking DP, Harcourt RG. 2016. Seeing it all: evaluating supervised machine learning methods for the classification of diverse otariid behaviours. PloS one 11:e0166898. DOI: 10.1371/journal.pone.0166898.

Leevy JL, Khoshgoftaar TM, Bauder RA, Seliya N. 2018. A survey on addressing high-class imbalance in big data. Journal of Big Data 5:1-30. DOI: 10.1186/s40537-018-0151-6.

Liaw A, Wiener M. 2002. Classification and regression by randomForest. $R$ news 2:18-22.

Lenth R, Singmann H, Love J, Buerkner P, Herve M. 2018. Emmeans: Estimated marginal means, aka least-squares means. R package version 1.1.3.

Lockyer C. 1984. Review of baleen whale (Mysticeti) reproduction and implications for management. Rep. int. Whal. Commn 6:27-50.

López LMM, Miller PJO, De Soto NA, Johnson M. 2015. Gait switches in deep-diving beaked whales: Biomechanical strategies for long-duration dives. Journal of Experimental Biology 218. DOI: $10.1242 /$ jeb.106013.

Morete ME, Freitas A, Engel MH, Pace III RM, Clapham PJ. 2003. A novel behavior observed in humpback whales on wintering grounds at Abrolhos Bank (Brazil). Marine Mammal Science 19:694-707.

Nathan R, Spiegel O, Fortmann-Roe S, Harel R, Wikelski M, Getz WM. 2012. Using tri-axial acceleration data to identify behavioral modes of free-ranging animals: general concepts and tools illustrated for griffon vultures. Journal of Experimental Biology 215:986-996. DOI: $10.1242 /$ jeb.058602.

Noad MJ, Cato DH. 2007. Swimming speeds of singing and non-singing humpback whales during migration. Marine Mammal Science 23:481-495. DOI: 10.1111/j.17487692.2007.02414.x.

Noren SR, Biedenbach G, Edwards EF. 2006. Ontogeny of swim performance and mechanics in bottlenose dolphins (Tursiops truncatus). Journal of Experimental Biology 209:4724-4731. DOI: $10.1242 /$ jeb.02566.

Noren SR, Edwards EF. 2011. Infant position in mother-calf dolphin pairs: formation locomotion with hydrodynamic benefits. Marine Ecology Progress Series 424:229-236. DOI: $10.3354 /$ meps 08986.

Oftedal OT. 1993. The adaptation of milk secretion to the constraints of fasting in bears, seals, and baleen whales. Journal of dairy science 76:3234-3246. DOI: 10.3168/jds.S00220302(93)77660-2.

Oftedal OT, Boness DJ, Tedman RA. 1987. The behavior, physiology, and anatomy of lactation in the pinnipedia. In: Current mammalogy. Springer, 175-245. DOI: 10.1007/978-1-47579909-5_6.

Pond CM. 1977. The significance of lactation in the evolution of mammals. Evolution 31:177199. DOI: $10.2307 / 2407556$.

Saloma A. 2018. Humpback whale (Megaptera novaeangliae) mother-calf interactions. Université d'Antananarivo \& Université Paris-Saclay.

Peer) reviewing PDF | (2021:10:66429:1:1:NEW 11 Jan 2022) 
973

974

975

976

977

978

979

980

981

982

983

984

985

986

987

988

989

990

991

992

993

994

995

996

997

998

999

1000

1001

1002

1003

1004

1005

1006

1007

1008

1009

1010

1011

1012

1013

1014

Shindo J, Yamada TK, Yoshimura K, Kageyama I. 2008. Morphology of the tongue in a newborn stejneger's beaked whale (Mesoplodon stejnegeri). Okajimas folia anatomica Japonica 84:121-124. DOI: 10.2535/ofaj.84.121.

Simon M, Johnson M, Madsen PT. 2012. Keeping momentum with a mouthful of water: behavior and kinematics of humpback whale lunge feeding. Journal of Experimental Biology 215:3786-3798. DOI: 10.1242/jeb.071092.

Slijper EJ. 1966. Functional morphology of the reproductive system in Cetacea. In: Whales, dolphins, and porpoises. 277-319. DOI: 10.1525/9780520321373-017.

Smith JM. 1977. Parental investment: A prospective analysis. Animal Behaviour 25:1-9. DOI: 10.1016/0003-3472(77)90062-8.

Steiger GH, Calambokidis J. 2000. Reproductive rates of humpback whales off California. Marine Mammal Science 16:220-239. DOI: 10.1111/j.1748-7692.2000.tb00914.x.

Stimpert AK, Mattila D, Nosal E-M, Au WWL. 2012. Tagging young humpback whale calves: methodology and diving behavior. Endangered Species Research 19:11-17. DOI: 10.3354/esr00456.

Tackaberry JE, Cade DE, Goldbogen JA, Wiley DN, Friedlaender AS, Stimpert AK. 2020. From a calf's perspective: humpback whale nursing behavior on two US feeding grounds. PeerJ 8:e8538. DOI: $10.7717 /$ peerj.8538.

Tennessen JB, Holt MM, Hanson MB, Emmons CK, Giles DA, Hogan JT. 2019. Kinematic signatures of prey capture from archival tags reveal sex differences in killer whale foraging activity. Journal of Experimental Biology 222. DOI: 10.1242/jeb.191874.

Trudelle L, Cerchio S, Zerbini AN, Geyer Y, Mayer F-X, Jung J-L, Hervé MR, Pous S, Sallée J$\mathrm{B}$, Rosenbaum HC, others. 2016. Influence of environmental parameters on movements and habitat utilization of humpback whales (Megaptera novaeangliae) in the Madagascar breeding ground. Royal Society open science 3:160616. DOI: 10.1098/rsos.160616.

Valletta JJ, Torney C, Kings M, Thornton A, Madden J. 2017. Applications of machine learning in animal behaviour studies. Animal Behaviour 124:203-220. DOI: 10.1016/j.anbehav.2016.12.005.

Vázquez Diosdado JA, Barker ZE, Hodges HR, Amory JR, Croft DP, Bell NJ, Codling EA. 2015. Classification of behaviour in housed dairy cows using an accelerometer-based activity monitoring system. Animal Biotelemetry 3:15. DOI: 10.1186/s40317-015-0045-8.

Videsen SKA, Bejder L, Johnson M, Madsen PT. 2017. High suckling rates and acoustic crypsis of humpback whale neonates maximise potential for mother--calf energy transfer. Functional Ecology 31:1561-1573. DOI: 10.1111/1365-2435.12871.

Wilson RP, White CR, Quintana F, Halsey LG, Liebsch N, Martin GR, Butler PJ. 2006. Moving towards acceleration for estimates of activity-specific metabolic rate in free-living animals: the case of the cormorant. Journal of Animal Ecology 75:1081-1090. DOI: 10.1111/j.13652656.2006.01127.x.

Zoidis AM, Lomac-MacNair KS. 2017. A note on suckling behavior and laterality in nursing humpback whale calves from underwater observations. Animals 7:51. DOI: 10.3390/ani7070051.

Peer) reviewing PDF | (2021:10:66429:1:1:NEW 11 Jan 2022) 


\section{Figure 1}

Screen capture of a video footage of humpback whale calf suckling on the right teat of its mother.

Photo credit: Isabelle Charrier.

Mother

Right mammary slit region

Calf's lower jaw

Calf's left eye

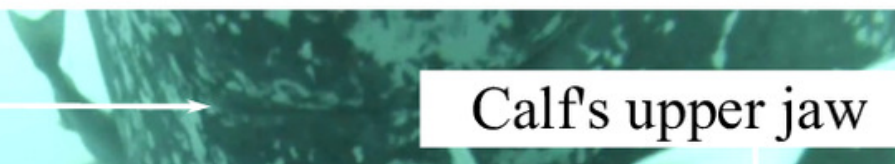

Calf's tongue 
Figure 2

Comparison of suckling events (18.8 s average duration) and non-suckling segments (20 $\mathrm{s}$ duration) with respect to activity phases.

Avg.: average. Mean and median are indicated by diamond marks and bold horizontal lines respectively. No ascent suckling has been observed.
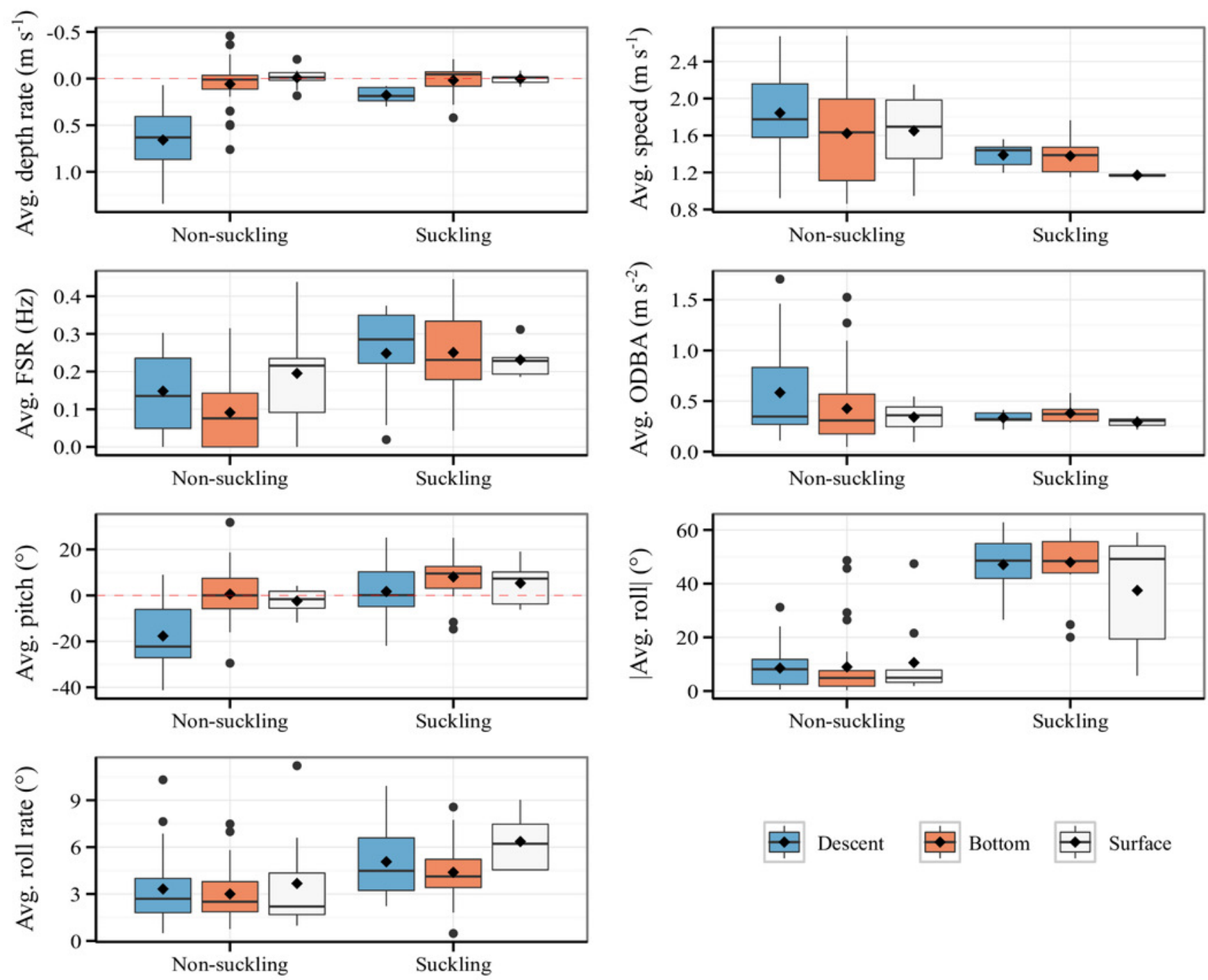

Descent 


\section{Figure 3}

Models' performance in automatically identifying suckling blocks.

The data included all three tag deployments on humpback whale calf. A 60:40 holdout training-testing split was used. (A) Sensitivity versus Precision plot of 12 models pre-selected using a Bayesian optimization approach. The symbols show the mean values and the SD. The models were pre-selected in the optimization procedure for each type of classifier over 30 runs. (B) Example of global results from automatic identification of suckling behaviour using the AdaBoostM1 model. (C) Example of results from automatic identification of suckling behaviour using the AdaBoostM1 model for Calf1. (D) Performance of the AdaBoostM1 model when the training set size is reduced. The symbols show the mean values and the SD (30 runs). 


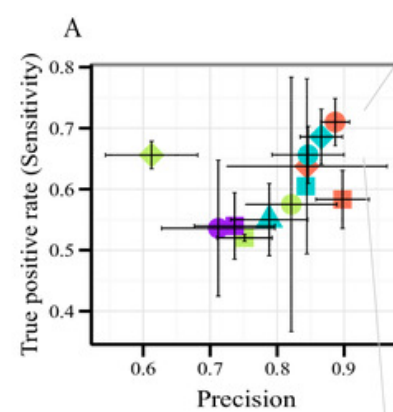

Ensemble: AdaBoostM1, 17 rum Fscore $=0.79 \pm 0.02$

Ensemble: Bag, 4 runs Fscore $=0.71 \pm 0.05$

Ensemble: GentleBoost, 9 runs

Fscore $-0.72 \pm 0.13$

KNN: Cityblock, 15 runs

Fscore $=0.74 \pm 0.03$

$\mathrm{KNN}$ : Cosine, 1 ru

Fscore $=0.70$

KNN: Minkowski, 12 runs Fscore $=0.77 \pm 0.03$

KNN: Euclidean, 2 runs Fscore $=0.65 \pm 0.02$

Decision tree: Deviance, 19 runs

Fscore $=0.60 \pm 0.06$

Decision tree: Gdi, 11 runs

Fscore $=0.62 \pm 0.03$

SVM: Gaussian, 26 runs
Fscore $-0.64 \pm 0.23$

SVM: Lincar, 2 num

Fscore $=0.61 \pm 0.01$

SVM: Polynomial, 2 runs

Fscore $=0.63 \pm 0.03$
B
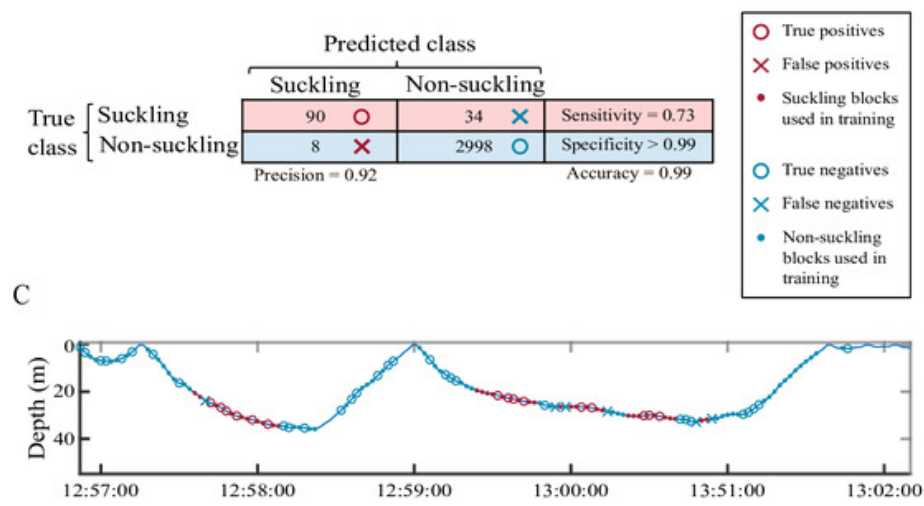

D

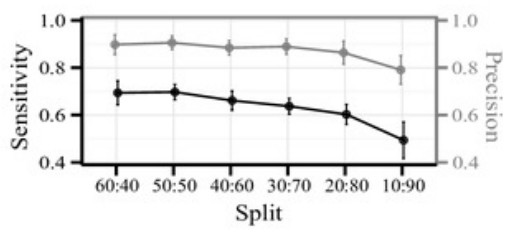




\section{Figure 4}

Sensitivity versus precision plot obtained from AdaBoostM1 model for suckling events using leave-one-out splits on data from three tag deployments on humpback whale calf.

In a leave-one-out split, data from two individuals were used for training while one individual is kept unseen for testing. The plot shows the classifier's performance when all features were included (open symbols) versus when the features that contributed most to the interindividual variation in terms of suckling were excluded (blue symbols). For comparison, mean results from 60:40 holdout split (purple symbol) and the worst scenario (red cross symbol) are showed.

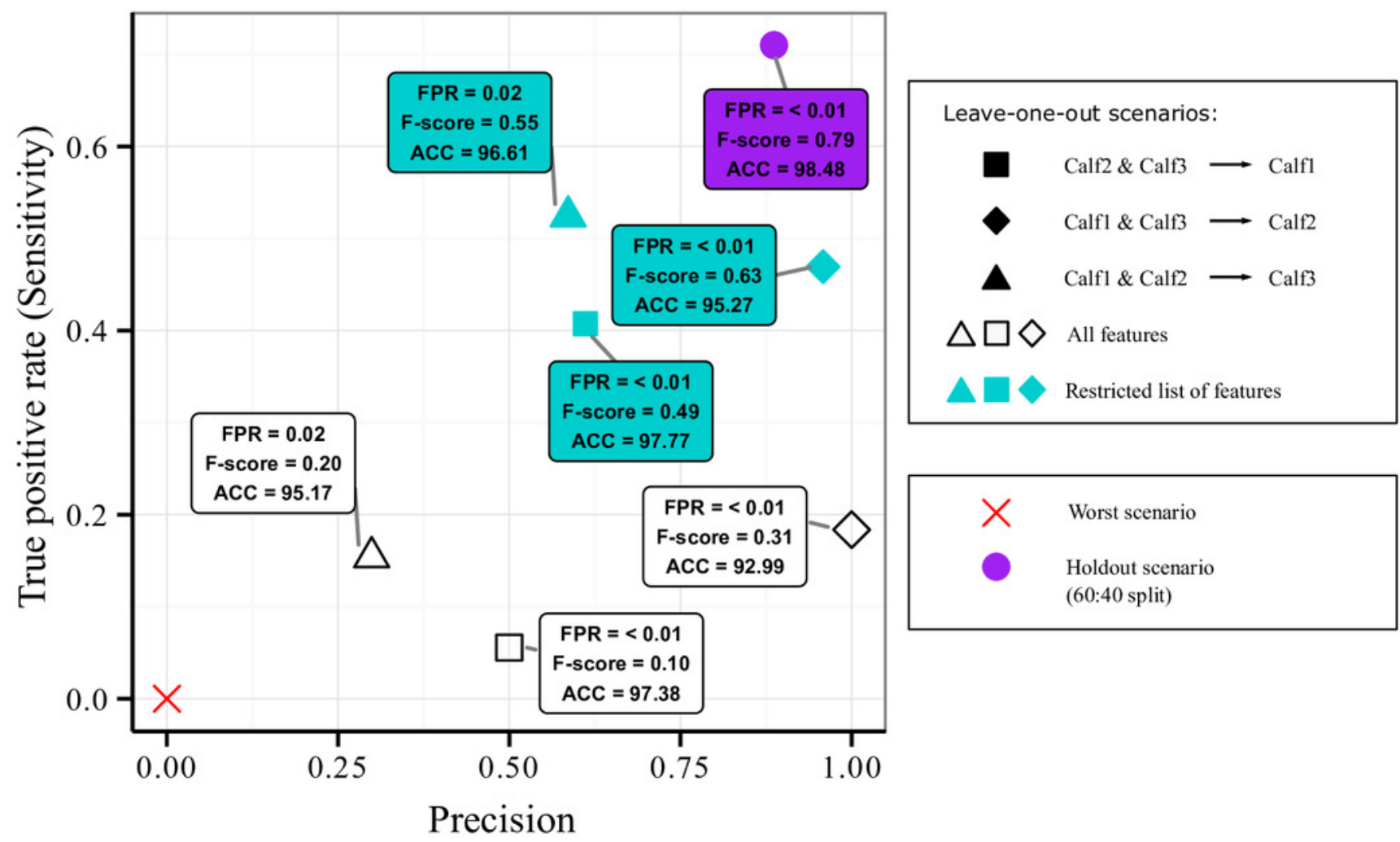




\section{Figure 5}

Analysis of the inter-individual difference between the suckling blocks of three humpback whale calves.

(A) Result of a Random Forest classification (confusion matrix) of the suckling blocks by individuals when all features were used. (B) Importance of the features in the classification by individuals. A sharp drop of the Gini index is noticeable after the 9th feature (red arrow). (C) Result of a Random Forest classification (confusion matrix) of the suckling blocks by individuals when the first 9 most important features were excluded. 
A
Predicted class

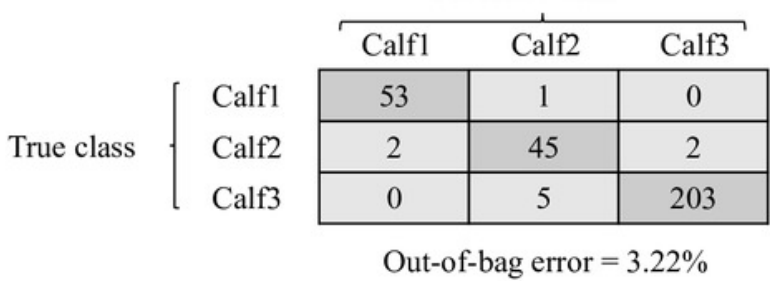

B

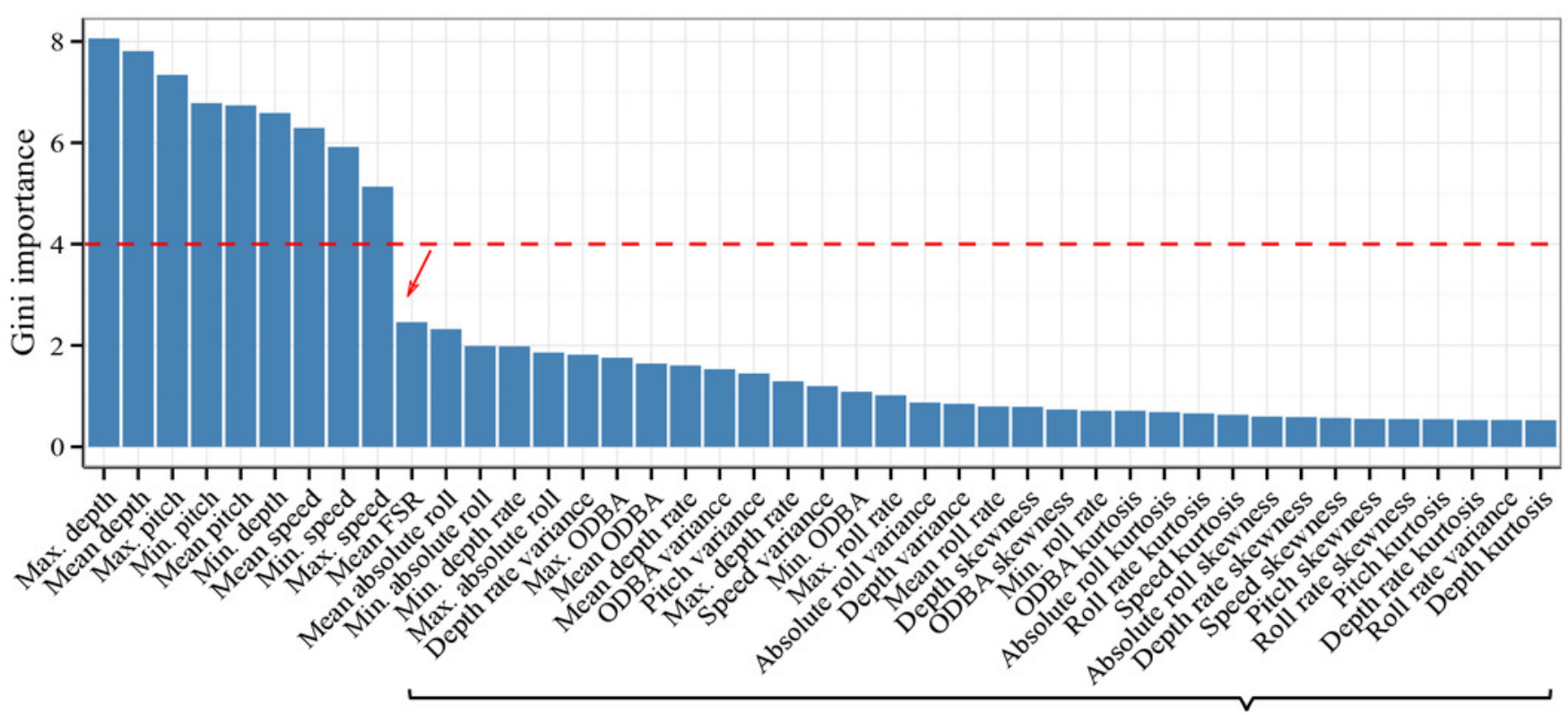

C

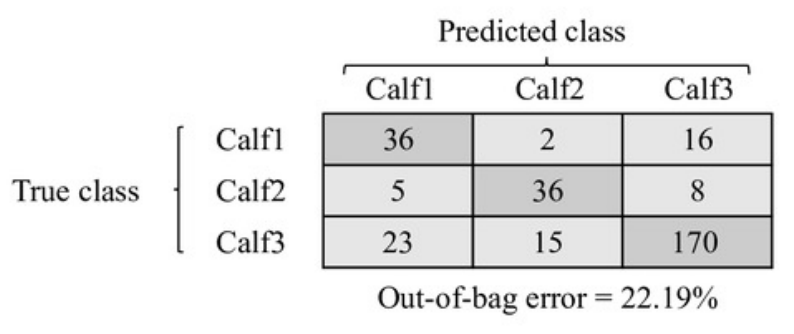




\section{Table $\mathbf{1}$ (on next page)}

Characteristics of all suckling events for each calf.

Date-times are presented in the format DD/MM/YYYY hhmm - hhmm and corresponds to deployment date, data start time, and data end time. Data start time also corresponds to tag attachment time. Data end time corresponds to tag detachment time, except for Calf3 since the data towards the end of the deployment on Calf3 was not usable due to lack of visibility on the video recording as the evening approached (the tag detached at 1845). The letters $\mathrm{R} / \mathrm{L}$ indicate the rolling sides of the calf as observed on the video recordings: Right/Left. Budget represents the proportion of time the animal was observed suckling. The remaining values are presented following the format mean $\pm S D(\min ; \max )$. Note the use of absolute value for roll to emphasize the roll deviation from zero (regardless of the side). No suckling events were observed during ascent phase of dives. 
Averages.

\begin{tabular}{|c|c|c|c|c|c|c|c|c|c|c|c|c|c|}
\hline \multirow[b]{2}{*}{ ID } & \multirow[b]{2}{*}{ Date-time } & \multirow[b]{2}{*}{ Occurrence } & \multirow[b]{2}{*}{$R / L$} & \multirow[b]{2}{*}{$\begin{array}{c}\text { Duration } \\
\text { (s) }\end{array}$} & \multirow[b]{2}{*}{$\begin{array}{c}\text { Budget } \\
(\%)\end{array}$} & \multicolumn{8}{|c|}{ ivera } \\
\hline & & & & & & $\begin{array}{l}\text { Depth } \\
\text { (m) }\end{array}$ & $\begin{array}{l}\text { Depth } \\
\text { rate } \\
\left(\mathrm{m} \mathrm{s}^{-1}\right)\end{array}$ & $\begin{array}{l}\text { Speed } \\
\left(\mathrm{m} \mathrm{s}^{-1}\right)\end{array}$ & $\begin{array}{l}\text { FSR } \\
(\mathrm{Hz})\end{array}$ & $\begin{array}{l}\text { Pitch } \\
\left(^{\circ}\right)\end{array}$ & $\begin{array}{c}\text { Roll } \mid \\
\left({ }^{\circ}\right)\end{array}$ & $\begin{array}{c}\text { Roll rate } \\
\left({ }^{\circ} \mathbf{s}^{-1}\right)\end{array}$ & $\begin{array}{c}\text { ODBA } \\
\left(\mathrm{m} \mathrm{s}^{-2}\right)\end{array}$ \\
\hline \multirow{2}{*}{ Calf1 } & \multirow{2}{*}{$\begin{array}{c}14 / 09 / 2018 \\
1134-1418\end{array}$} & $\begin{array}{c}\text { During dive } \\
\text { (descent) } \\
N=2 \\
\end{array}$ & $1 / 1$ & $\begin{array}{c}23.3 \pm 1.5 \\
(22.2 ; \\
24.3) \\
\end{array}$ & 0.5 & $\begin{array}{c}24.6 \pm 3.3 \\
(22.3 ; \\
26.9) \\
\end{array}$ & $\begin{array}{c}0.2 \pm 0.1 \\
(0.1 ; 0.2)\end{array}$ & $\begin{array}{c}1.5 \pm 0.1 \\
(1.4 ; 1.5)\end{array}$ & $\begin{array}{c}0.3 \\
(0.3 ; 0.3)\end{array}$ & $\begin{array}{c}-13 \pm 12 \\
(-22 ;-5) \\
\end{array}$ & $\begin{array}{c}39 \pm 13 \\
(30 ; 48) \\
\end{array}$ & $\begin{array}{c}3.6 \pm 1.9 \\
(2.2 ; 4.9) \\
\end{array}$ & $\begin{array}{c}0.4 \\
(0.3 ; 0.4) \\
\end{array}$ \\
\hline & & $\begin{array}{c}\text { During dive } \\
\text { (bottom) } \\
N=3\end{array}$ & $2 / 1$ & $\begin{array}{c}22.2 \pm 12.8 \\
(8.8 ; \\
34.2) \\
\end{array}$ & 0.7 & $\begin{array}{c}30.6 \pm 1.2 \\
(29.3 ; \\
31.8) \\
\end{array}$ & $\begin{array}{c}0.1 \pm 0.3 \\
(-0.2 ; \\
0.4) \\
\end{array}$ & $\begin{array}{c}1.4 \pm 0.1 \\
(1.4 ; 1.5) \\
\end{array}$ & $\begin{array}{c}0.2 \\
(0.1 ; 0.2)\end{array}$ & $\begin{array}{c}-5 \pm 14 \\
(-15 ; 12)\end{array}$ & $\begin{array}{c}37 \pm 15 \\
(20 ; 49) \\
\end{array}$ & $\begin{array}{c}4.1 \pm 0.8 \\
(3.5 ; 5.1)\end{array}$ & $\begin{array}{c}0.4 \pm 0.1 \\
(0.3 ; 0.5) \\
\end{array}$ \\
\hline \multirow{2}{*}{ Calf2 } & \multirow{2}{*}{$\begin{array}{c}06 / 08 / 2019 \\
1057-1234\end{array}$} & $\begin{array}{c}\text { During dive } \\
\text { (descent) } \\
N=1 \\
\end{array}$ & $0 / 1$ & 44.9 & 0.8 & 22.2 & 0.1 & 1.6 & 0.1 & 25 & 63 & 3.5 & 0.4 \\
\hline & & $\begin{array}{c}\text { During dive } \\
\text { (bottom) } \\
N=3 \\
\end{array}$ & $3 / 0$ & $\begin{array}{c}18.4 \pm 12.3 \\
(6.2 ; \\
30.9) \\
\end{array}$ & 0.9 & $\begin{array}{c}26.1 \pm 6.7 \\
(19.3 ; \\
32.6) \\
\end{array}$ & $\begin{array}{c}0 \pm 0.2 \\
(-0.1 ; \\
0.3) \\
\end{array}$ & $\begin{array}{c}1.7 \pm 0.1 \\
(1.6 ; 1.8)\end{array}$ & $\begin{array}{c}0.2 \\
(0.2 ; 0.2)\end{array}$ & $\begin{array}{c}11 \pm 7 \\
(3 ; 16)\end{array}$ & $\begin{array}{c}47 \pm 3 \\
(44 ; 48)\end{array}$ & $\begin{array}{c}3.7 \pm 0.9 \\
(3.1 ; 4.8)\end{array}$ & $\begin{array}{c}0.5 \pm 0.1 \\
(0.5 ; 0.6)\end{array}$ \\
\hline \multirow{3}{*}{ Calf3 } & \multirow{3}{*}{$\begin{array}{c}09 / 08 / 2019 \\
1003-1802\end{array}$} & $\begin{array}{c}\text { During dive } \\
\text { (descent) } \\
N=6\end{array}$ & $2 / 4$ & $\begin{array}{c}10.9 \pm 4.2 \\
(4.8 ; \\
15.6) \\
\end{array}$ & 0.3 & $\begin{array}{c}12.4 \pm 3.4 \\
(8.9 ; \\
17.8) \\
\end{array}$ & $\begin{array}{c}0.2 \pm 0.1 \\
(0.1 ; 0.3) \\
\end{array}$ & $\begin{array}{c}1.3 \pm 0.1 \\
(1.2 ; 1.5)\end{array}$ & $\begin{array}{c}0.3 \pm 0.1 \\
(0 ; 0.4)\end{array}$ & $\begin{array}{c}3 \pm 10 \\
(-10 ; 19)\end{array}$ & $\begin{array}{c}47 \pm 12 \\
(27 ; 63)\end{array}$ & $\begin{array}{c}5.8 \pm 2.7 \\
(3.1 ; 9.9)\end{array}$ & $\begin{array}{c}0.3 \pm 0.1 \\
(0.2 ; 0.4)\end{array}$ \\
\hline & & $\begin{array}{c}\text { During dive } \\
\text { (bottom) } \\
N=14\end{array}$ & $8 / 6$ & $\begin{array}{c}18.9 \pm 4.6 \\
(11.2 ; \\
25.5)\end{array}$ & 1.1 & $\begin{array}{c}16.8 \pm 3.2 \\
(11.1 ; \\
21.1)\end{array}$ & $\begin{array}{c}0 \pm 0.1 \\
(-0.1 ; \\
0.3)\end{array}$ & $\begin{array}{c}1.3 \pm 0.2 \\
(1.1 ; 1.7)\end{array}$ & $\begin{array}{l}0.3 \pm 0.1 \\
(0 ; 0.4)\end{array}$ & $\begin{array}{c}10 \pm 7 \\
(0 ; 25)\end{array}$ & $\begin{array}{c}51 \pm 9 \\
(25 ; 61)\end{array}$ & $\begin{array}{c}4.6 \pm 2.1 \\
(0.5 ; 8.6)\end{array}$ & $\begin{array}{c}0.3 \pm 0.1 \\
(0.3 ; 0.4)\end{array}$ \\
\hline & & $\begin{array}{c}\text { At surface } \\
N=5\end{array}$ & $3 / 2$ & $\begin{array}{c}19.6 \pm 10 \\
(7.9 ; 35)\end{array}$ & 0.4 & $\begin{array}{l}6.2 \pm 2.7 \\
(2 ; 8.4)\end{array}$ & $\begin{array}{c}0 \pm 0.1 \\
(-0.1 ; \\
0.1)\end{array}$ & $\begin{array}{c}1.2 \\
(1.2 ; 1.2)\end{array}$ & $\begin{array}{c}0.2 \pm 0.1 \\
(0.2 ; 0.3)\end{array}$ & $\begin{array}{c}5 \pm 10 \\
(-6 ; 19)\end{array}$ & $\begin{array}{c}37 \pm 24 \\
(6 ; 59)\end{array}$ & $\begin{array}{l}6.4 \pm 1.9 \\
(4.5 ; 9)\end{array}$ & $\begin{array}{c}0.3 \pm 0.1 \\
(0.2 ; 0.4)\end{array}$ \\
\hline
\end{tabular}

